\title{
Numerical Simulations of Immiscible Fluid Clusters
}

\author{
Robert Nürnberg ${ }^{\dagger}$
}

\begin{abstract}
We present a phase field model for multi-component surface diffusion, which can be used to describe the evolution of clusters of immiscible fluids such as soap bubble clusters. The model is given by a Cahn-Hilliard system with a non-smooth obstacle free energy and a degenerate mobility matrix. On stating the considered finite element approximation, we describe the iterative solver used to solve the nonlinear discrete system at each time step and present several numerical experiments for $N=$ $3,4,5$ and 6 components in two and three space dimensions, including simulations with topological changes.
\end{abstract}

Key words. multi-component systems, degenerate parabolic systems of fourth order, phase field model, finite element method, surface diffusion, soap bubbles

AMS subject classifications. 65M60, 35K55, 35K65, 82C26, 65M50, 82C24

\section{Introduction}

In classical models of fluid systems, interfaces between two fluids are treated as infinitely thin, or sharp, and are endowed with properties such as surface tension. In this paper, we consider a vector valued Cahn-Hilliard equation as a phase field model (also called diffuse interface model) for the evolution of such fluid clusters in $\mathbb{R}^{d}$, with $d=2,3$. Originally, Cahn-Hilliard systems were studied in order to model phase separation and coarsening in multi-component systems such as alloys or polymers, see [39, 19]. However, it was established in [27] via formal asymptotics that a singular limit of degenerate Cahn-Hilliard systems gives rise to a geometric evolution equation, the so-called multi-component surface diffusion. This evolution law is of particular interest, because it can be used as a simplified model for the evolution of immiscible fluid clusters, such as soap bubbles. In particular, steady state solutions of the flow (which typically consist of constant mean curvature segments that meet at triple junctions and, for $d=3$ and $N \geq 4$, quadruple junction points) are the natural candidates for area minimizing soap bubble constellations for fixed given volume fractions. We note that there is great interest in these bubble constellations, with

\footnotetext{
${ }^{\dagger}$ Department of Mathematics, Imperial College London, London, SW7 2AZ, UK
} 
many open questions still remaining. See e.g. [43, 23, 34, 37, 28, 1, 42, 45, 38, 29, 22, 44] and the references therein. In this paper, we will often not make the distinction between the multi-component system in the phase field model and the analogue in the sharp interface limit, i.e. we will refer to a fixed enclosed volume/bubble also as a "component" and vice versa.

We now present the phase field model that we will consider in this paper, where we largely follow the introduction of the degenerate Cahn-Hilliard system in [21]. We consider a cluster of immiscible fluids $F_{1}, \ldots, F_{N}$ in $\mathbb{R}^{d}$, with $F_{1}$ the ambient fluid. We introduce the interfacial parameter $\gamma \in \mathbb{R}_{>0}$ and the conserved vector-valued order parameter $\mathbf{u}_{\gamma}(\cdot, t)=$ $\left(u_{\gamma}^{1}, \ldots, u_{\gamma}^{N}\right)^{T} \in \mathbb{R}^{N}$, where at any time $t \in[0, T]$ we denote by $u_{\gamma}^{n}(\cdot, t)$ the fractional concentration of the $n^{\text {th }}$ fluid/component. Physically meaningful values for the order parameter $\mathbf{u}_{\gamma}$ have nonnegative entries and satisfy $\sum_{n=1}^{N} u_{\gamma}^{n}=1$. It is therefore convenient to define the Gibbs simplex

$$
\mathbb{Q}^{N}:=\left\{\boldsymbol{\zeta} \in \mathbb{R}^{N}: \sum_{n=1}^{N} \zeta^{n}=1, \boldsymbol{\zeta} \geq \mathbf{0}\right\} \subset \mathbb{R}^{N}
$$

as the set of meaningful values for $\mathbf{u}_{\gamma}$. Hence the pure phases can be identified by $\mathbf{u}_{\gamma}=\boldsymbol{e}_{n}$, where $e_{n}^{p}=\delta_{n p}, p=1 \rightarrow N$, with $\delta_{n p}$ denoting the Kronecker delta. Next we introduce the free energy $\mathcal{E}_{\gamma}$, which is of the Ginzburg-Landau form

$$
\mathcal{E}_{\gamma}\left(\mathbf{u}_{\gamma}\right):=\int_{\Omega}\left(\frac{\gamma}{2}\left|\nabla \mathbf{u}_{\gamma}\right|^{2}+\frac{1}{\gamma} \Psi\left(\mathbf{u}_{\gamma}\right)\right) \mathrm{d} x
$$

where $\Omega$ is a bounded domain in $\mathbb{R}^{d}, d=2,3$. The homogeneous free energy density $\Psi$ is given by

$$
\Psi\left(\mathbf{u}_{\gamma}\right):=\Psi_{0}\left(\mathbf{u}_{\gamma}\right)-\frac{1}{2} \mathbf{u}_{\gamma}^{T} A \mathbf{u}_{\gamma},
$$

where $A$ is a constant symmetric $N \times N$ matrix that describes the surface energy densities between different fluids/components. A typical choice is

$$
A=\mathrm{id}_{N}-\mathbf{1} \mathbf{1}^{T}
$$

which is the case when all the surface energy densities are the same. Here $\mathbf{1}=(1, \ldots, 1)^{T} \in$ $\mathbb{R}^{N}$ and $\operatorname{id}_{N}$ is the identity matrix in $\mathbb{R}^{N}$. Finally, the term $\Psi_{0}$ represents the obstacle free energy

$$
\Psi_{0}\left(\mathbf{u}_{\gamma}\right):= \begin{cases}0 & \text { if } \mathbf{u}_{\gamma} \in \mathbb{Q}^{N}, \\ \infty & \text { if } \mathbf{u}_{\gamma} \in \mathbb{R}^{N} \backslash \mathbb{Q}^{N},\end{cases}
$$

which forces $\mathbf{u}_{\gamma}$ to take values inside the Gibbs simplex $\mathbb{Q}^{N}$. Moreover, we will always assume that $A_{n p} \leq 0$, with equality if and only if $n=p$, so that the free energy density has $N$ global minima $\Psi\left(\boldsymbol{e}_{n}\right)=0, n=1 \rightarrow N$, at the vertices of the Gibbs simplex.

In order to formulate the evolution equation for the order parameter $\mathbf{u}_{\gamma}$, we introduce the chemical potential $\mathbf{w}_{\gamma}=\left(w_{\gamma}^{1}, \ldots, w_{\gamma}^{N}\right)^{T}$, where $w_{\gamma}^{n}$ is the variational derivative of $\mathcal{E}_{\gamma}$ with respect to the $n^{\text {th }}$ component, i.e. formally

$$
\mathbf{w}_{\gamma}=-\gamma \Delta \mathbf{u}_{\gamma}+\gamma^{-1} \mathbf{D} \Psi\left(\mathbf{u}_{\gamma}\right),
$$


where $\left\{\mathbf{D} \Psi\left(\mathbf{u}_{\gamma}\right)\right\}^{n}:=\frac{\partial \Psi}{\partial u^{n}}\left(\mathbf{u}_{\gamma}\right)$. We note that as $\Psi$, with $\Psi_{0}$ given by (1.5), is nondifferentiable, the above identity only holds where $\mathbf{u}_{\gamma} \in \mathbb{Q}^{N} \backslash \partial \mathbb{Q}^{N}$. In general, we need to solve a variational inequality in order to obtain $\mathbf{w}_{\gamma}$, see $(1.11 \mathrm{~b})$ below.

The evolution equations for $\mathbf{u}_{\gamma}$ are then given by

$$
\gamma \frac{\partial}{\partial t} \mathbf{u}_{\gamma}=\nabla \cdot\left(L\left(\mathbf{u}_{\gamma}\right) \nabla \mathbf{w}_{\gamma}\right)
$$

where $L$ is a symmetric $N \times N$ mobility matrix and in this paper is taken of the form

$$
\{L(\boldsymbol{\zeta})\}_{n p}=\zeta^{n}\left(\delta_{n p}-\zeta^{p}\right) \quad \zeta \in \mathbb{Q}^{N} .
$$

More general choices for the mobility matrix are possible, for example

$$
\{L(\boldsymbol{\zeta})\}_{n p}=l^{n}\left(\zeta^{n}\right)\left(\delta_{n p}-\left[\mathbf{1}^{T} \boldsymbol{l}(\boldsymbol{\zeta})\right]^{-1} l^{p}\left(\zeta^{p}\right)\right),
$$

where $\boldsymbol{l}(\boldsymbol{\zeta}):=\left(l^{1}\left(\zeta^{1}\right), \ldots, l^{N}\left(\zeta^{N}\right)\right)^{T}$ and the bare mobilities are continuous functions $l^{n} \in$ $C\left([0,1] ; \mathbb{R}_{\geq 0}\right), n=1 \rightarrow N$. Clearly, the choice (1.8) corresponds to setting $l^{n}(s)=s$, $n=1 \rightarrow N$, in (1.9). We note that $L(\boldsymbol{\zeta})$ is symmetric positive semi-definite and that $L(\boldsymbol{\zeta}) \mathbf{1}=\mathbf{0}$ for any $\boldsymbol{\zeta} \in \mathbb{Q}^{N}$. The latter implies, on noting (1.7), that $\gamma \frac{\partial}{\partial t} \sum_{n=1}^{N} u_{\gamma}^{n}=$ $\gamma \frac{\partial}{\partial t}\left(\mathbf{1}^{T} \mathbf{u}_{\gamma}\right)=0$, which means that (1.7) is always solved on the hyperplane

$$
\mathcal{H}^{N}:=\left\{\boldsymbol{\zeta} \in \mathbb{R}^{N}: \mathbf{1}^{T} \boldsymbol{\zeta}=1\right\}
$$

As the zero eigenvector $\mathbf{1}$ of the mobility matrix $L$ is perpendicular to the corresponding tangent space, this degeneracy does not affect the analysis, see [21]. However, the matrix $L$ degenerates further on the boundary $\partial \mathbb{Q}^{N}$ of the Gibbs simplex, i.e. $\operatorname{rank} L(\boldsymbol{\zeta}) \leq N-2$ for $\zeta \in \partial \mathbb{Q}^{N}$. Altogether this means that (1.7) is a fourth-order degenerate parabolic system of partial differential equations, also called degenerate Cahn-Hilliard system. This degeneracy makes the analysis and numerical treatment of (1.7) particularly difficult.

Before we proceed, we complement the equations (1.7) and (1.6) with the appropriate boundary and initial conditions and state precisely the problem we seek to solve for a fixed given $\gamma>0$.

$\left(\mathrm{P}_{\gamma}\right)$ Find functions $\left\{\mathbf{u}_{\gamma}, \mathbf{w}_{\gamma}\right\}: \Omega \times[0, T] \rightarrow \mathbb{Q}^{N} \times \mathbb{R}^{N}$ such that

$$
\begin{aligned}
\gamma \frac{\partial}{\partial t} \mathbf{u}_{\gamma}-\nabla \cdot\left(L\left(\mathbf{u}_{\gamma}\right) \nabla \mathbf{w}_{\gamma}\right) & =0 & & \text { in } \Omega_{T}, \\
\left(-\gamma \Delta \mathbf{u}_{\gamma}-\gamma^{-1} A \mathbf{u}_{\gamma}-\mathbf{w}_{\gamma}\right)^{T}\left(\boldsymbol{\eta}-\mathbf{u}_{\gamma}\right) & \geq 0 \quad \forall \boldsymbol{\eta} \in \mathbb{Q}^{N} & & \text { in } \Omega_{T}, \\
\frac{\partial}{\partial \nu} \mathbf{u}_{\gamma}=L\left(\mathbf{u}_{\gamma}\right) \frac{\partial}{\partial \nu} \mathbf{w}_{\gamma} & =\mathbf{0} & & \text { on } \partial \Omega \times \\
\mathbf{u}_{\gamma}(x, 0) & =\mathbf{u}_{\gamma}^{0}(x) \in \mathbb{Q}^{N} & & \forall x \in \Omega,
\end{aligned}
$$

where $T>0$ is a fixed positive time, $\Omega_{T}:=\Omega \times(0, T]$ and $\nu$ is the outer normal to the boundary $\partial \Omega$.

Existence of weak solutions to the degenerate Cahn-Hilliard system (1.11a-d) has been shown in $[20,21]$. A numerical approximation to $(1.11 \mathrm{a}-\mathrm{d})$ in the context of multicomponent phase separation was considered in [4], where in addition a convergence proof for the introduced finite element approximation was given for $d=1$. 
We note that it immediately follows from $(1.11 \mathrm{a}-\mathrm{d})$ that

$$
\frac{\mathrm{d}}{\mathrm{d} t} \int_{\Omega} \mathbf{u}_{\gamma} \mathrm{d} x=\mathbf{0}
$$

i.e. the fractional mass/volume concentrations are preserved. Moreover we see from (1.2) with e.g. (1.4), that it is desirable for $\mathbf{u}_{\gamma}$ to partition $\Omega$ into pure phases $\left\{\mathbf{u}_{\gamma}=\boldsymbol{e}_{n}\right\}$, $n=1 \rightarrow N$, with smooth diffuse interfacial layers between them, whose thickness is proportional to $\gamma$. On combining the techniques in $[27,13]$, see also $[2,11]$, it is possible to show that when the interfacial parameter $\gamma \rightarrow 0$, the system $(1.11 \mathrm{a}-\mathrm{d})$ approximates a sharp interface model of multi-component surface diffusion. The model can be used as a simplified approximation of the evolution of immiscible fluid clusters, such as soap bubbles, where gravitational and inertial effects are negligible. In particular, the limiting sharp interface model yields motion by minus the Laplacian of mean curvature for each of the involved interfaces. At triple junctions Young's law, a no-flux condition and a continuity condition for the chemical potentials have to hold. See e.g. [6, 8] for a detailed description of the sharp interface evolution equations. Note that Young's law in the equal surface energy density case (when $A$ is given by (1.4)) yields a $120^{\circ}$ triple junction angle condition, as well as a $\varphi \approx 109^{\circ}$ angle condition at quadruple junction points in $\mathbb{R}^{3}$, where $\cos \varphi=-\frac{1}{3}$. These angles are well-known in the theory of minimal surfaces, see e.g. [30]. We remark that the formal asymptotics for $\left(\mathrm{P}_{\gamma}\right)$ with (1.4) yield that the interface thickness of the diffuse interfaces is approximately $\gamma \pi$. We refer to $[2,12,26]$ and the forthcoming paper [8] for more details. In particular, it can be shown that, as $\gamma \rightarrow 0, \mathcal{E}_{\gamma}$ leads to a sharp interface free energy of the form

$$
E=\sum_{\substack{n, p=1 \\ n<p}}^{N} \varsigma_{n p}\left|\Gamma_{n p}\right|,
$$

where $\Gamma_{n p} \subset \mathbb{R}^{d}$ denotes the not necessarily connected and possibly empty interface separating phases $n$ and $p$, with surface energy densities

$$
\varsigma_{n p}=2 \inf _{\boldsymbol{q}} \int_{-1}^{1}\left|\boldsymbol{q}^{\prime}(s)\right| \sqrt{\frac{1}{2} \Psi(\boldsymbol{q}(s))} \mathrm{d} s, \quad n, p \in\{1, \ldots, N\},
$$

where the infimum is over all $\boldsymbol{q} \in C^{1}\left([-1,1], \mathbb{R}^{N}\right)$ with $\boldsymbol{q}(-1)=\boldsymbol{e}_{n}$ and $\boldsymbol{q}(1)=\boldsymbol{e}_{p}$.

The paper is organized as follows. In Section 2 we state the finite element approximation of $\left(\mathrm{P}_{\gamma}\right)$ that we employ in this paper and introduce an efficient and novel solution method for the discrete nonlinear systems of equations that need to be solved at each time level. In Section 3 we present numerous numerical results for the evolution of soap bubbles in $\mathbb{R}^{2}$ and $\mathbb{R}^{3}$. Of particular interest are the double bubble shapes and their stability; i.e. $N=3$. We note that the double bubble conjecture for $d=3$ has been shown only relatively recently in [31], see also [23] for the case $d=2$. The conjecture asserts that if $V_{1}$ and $V_{2}$ are two given volumes, then the standard double bubble has smallest possible surface area among all the surfaces enclosing and separating these two volumes. Here the standard double bubble is made up of three pieces of round spheres, 
meeting along a common circle at an angle of 120 degrees. See Figure 11 for an example for a standard double bubble and Figure 13 for a nonstandard one. The corresponding conjecture for a triple bubble, i.e. $N=4$, is still open for $d=3$, while the proof for the planar triple bubble, $d=2$, has recently been given in [46]. Remarkably, for $N \geq 5$ the corresponding results are still only conjectures, even for $d=2$, as was reported e.g. in $[15]$.

Existing approaches for the numerical approximation of soap bubbles include the well known Surface Evolver by Brakke, [10], where a direct parameterization and a gradient descent method is used in order to find the surface area minimizer for a given constellation of volumes. We note that the Surface Evolver has recently been used to numerically study large soap bubble clusters in two and three space dimensions, see e.g. [18, 17, 33, 16]. A level set approach for the simulation of the evolution of soap bubbles has been considered in [47]. However, the numerical results obtained there appear to be inaccurate, in that the steady state solutions reported there seem to differ from the true equilibrium solutions; see Section 3 for details. In addition, a direct parametric finite element approximation of multi-component surface diffusion for $d=2$, also called surface diffusion of curve networks, was recently proposed in [6]. There a novel variational formulation of geometric evolution equations for curves and curve networks is introduced, which leads to an unconditionally stable parametric finite element approximation. The highly nontrivial extension of this approach to $d=3$ is the subject of current research. Of course, the parametric approach fails for evolutions with topological changes, such as the merging of two bubbles. Numerical results for $N$-component Cahn-Hilliard systems, including the evolution of triple junctions in ternary systems, have been presented in $[4,35]$, while $N$-component Allan-Cahn systems with volume constraints have been considered in [25]. Here we recall that Allan-Cahn systems arise as the natural $L^{2}$ gradient flow of the energy (1.2), leading to a second-order system of parabolic equations. Enforcing volume preservation with the help of some side constraints allows the authors of [25] to numerically study equilibrium constellations of bubble clusters. Finally, an alternative finite element approximation of the phase field model $\left(\mathrm{P}_{\gamma}\right)$, for which it is possible to prove convergence in space dimensions $d=2$ and $d=3$, will be given in the forthcoming article [8]. To our knowledge, the numerical results presented in this paper, see Section 3, are the first simulations in the literature of multi-component surface diffusion in $\mathbb{R}^{3}$, and the first such simulations in $\mathbb{R}^{2}$ of evolutions that feature changes in topology in nontrivial domains.

\section{Approximation and iterative solver}

We now consider a finite element approximation of the phase field model $\left(\mathrm{P}_{\gamma}\right)$. To this end, we recall the finite element approximation from [4]. Let $\Omega$ be a polyhedral domain and let $\left\{\mathcal{T}^{h}\right\}_{h>0}$ be a family of partitionings of $\Omega$ into disjoint open simplices $\sigma$ with $h_{\sigma}:=\operatorname{diam}(\sigma)$ and $h:=\max _{\sigma \in \mathcal{T}^{h}} h_{\sigma}$, so that $\bar{\Omega}=\cup_{\sigma \in \mathcal{T}^{h}} \bar{\sigma}$. Associated with $\mathcal{T}^{h}$ is the finite element space

$$
S^{h}:=\left\{\chi \in C(\bar{\Omega}):\left.\chi\right|_{\sigma} \text { is linear } \forall \sigma \in \mathcal{T}^{h}\right\}
$$


We extend this definition to vector functions, i.e. $\boldsymbol{\chi} \in \boldsymbol{S}^{h} \Rightarrow \chi^{n} \in S^{h}, n=1 \rightarrow N$. Let $J$ be the set of nodes of $\mathcal{T}^{h}$ and let $\left\{p_{j}\right\}_{j \in J}$ be the coordinates of these nodes. Let $\left\{\chi_{j}\right\}_{j \in J}$ be the standard basis functions for $S^{h}$; that is $\chi_{j} \in S^{h}$ and $\chi_{j}\left(p_{i}\right)=\delta_{i j}$ for all $i, j \in J$.

We introduce $\pi^{h}: C(\bar{\Omega}) \rightarrow S^{h}$, the interpolation operator, such that $\pi^{h} \eta\left(p_{j}\right)=\eta\left(p_{j}\right)$ for all $j \in J$. A discrete semi-inner product on $C(\bar{\Omega})$ is defined by

$$
\left(\eta_{1}, \eta_{2}\right)^{h}:=\left(\pi^{h}\left[\eta_{1} \eta_{2}\right], 1\right),
$$

where here and throughout $(\cdot, \cdot)$ denotes the standard $L^{2}$ inner product over $\Omega$. We extend naturally the above definitions to vector functions; e.g. $\boldsymbol{\pi}^{h}: \boldsymbol{C}(\bar{\Omega}) \rightarrow \boldsymbol{S}^{h}$ with $\left\{\boldsymbol{\pi}^{h} \boldsymbol{\eta}\right\}^{n}:=\pi^{h} \eta^{n}$. For later purposes, we also introduce the following convex subsets of $\boldsymbol{S}^{h}$ :

$$
\boldsymbol{S}_{\geq 0}^{h}:=\left\{\boldsymbol{\chi} \in \boldsymbol{S}^{h}: \boldsymbol{\chi}\left(p_{j}\right) \geq \mathbf{0} \quad \forall j \in J\right\}, \quad \boldsymbol{K}^{h}:=\left\{\boldsymbol{\chi} \in \boldsymbol{S}_{\geq 0}^{h}: \mathbf{1}^{T} \boldsymbol{\chi}\left(p_{j}\right)=1 \quad \forall j \in J\right\} .
$$

Following [4], we now introduce a regularized version of our chosen degenerate mobility matrix (1.8). To this end, for any $\varepsilon>0$, let

$$
l_{\varepsilon}^{n}(s):=s+\varepsilon \quad s \in[0,1], \quad n=1 \rightarrow N,
$$

and then, similarly to (1.9), let the regularized mobility matrix $L_{\varepsilon}$ be defined by

$$
\left\{L_{\varepsilon}(\boldsymbol{\zeta})\right\}_{n p}=l_{\varepsilon}^{n}\left(\zeta^{n}\right)\left(\delta_{n p}-\left[\mathbf{1}^{T} \boldsymbol{l}_{\varepsilon}(\boldsymbol{\zeta})\right]^{-1} l_{\varepsilon}^{p}\left(\zeta^{p}\right)\right) .
$$

Moreover, for our numerical approximation of problem $\left(\mathrm{P}_{\gamma}\right)$ we split

$$
A \equiv A^{+}+A^{-}, \quad \text { where } A^{+(-)} \text {is symmetric positive (negative) semi-definite. }
$$

In addition, let $0 \equiv t_{0}<t_{1}<\cdots t_{K-1}<t_{K} \equiv T$ be a partitioning of $[0, T]$ into possibly variable time steps $\tau_{k}:=t_{k}-t_{k-1}, k=1 \rightarrow K$. Let $\tau:=\max _{k=1 \rightarrow K} \tau_{k}$. For a fixed $\gamma>0$, we then consider the following fully practical finite element approximation of $\left(\mathrm{P}_{\gamma}\right)$ :

For $k \geq 1$, find $\left\{\boldsymbol{U}_{\varepsilon}^{k}, \boldsymbol{W}_{\varepsilon}^{k}\right\} \in \boldsymbol{S}_{\geq 0}^{h} \times \boldsymbol{S}^{h}$ such that for all $\boldsymbol{\eta} \in \boldsymbol{S}^{h}$ and $\boldsymbol{\chi} \in \boldsymbol{S}_{\geq 0}^{h}$

$$
\begin{gathered}
\gamma\left(\frac{\boldsymbol{U}_{\varepsilon}^{k}-\boldsymbol{U}_{\varepsilon}^{k-1}}{\tau_{k}}, \boldsymbol{\eta}\right)^{h}+\left(L_{\varepsilon}\left(\boldsymbol{U}_{\varepsilon}^{k-1}\right) \nabla \boldsymbol{W}_{\varepsilon}^{k}, \nabla \boldsymbol{\eta}\right)=0, \\
\gamma\left(\nabla \boldsymbol{U}_{\varepsilon}^{k}, \nabla\left(\boldsymbol{\chi}-\boldsymbol{U}_{\varepsilon}^{k}\right)\right)-\left(\gamma^{-1} A^{-} \boldsymbol{U}_{\varepsilon}^{k}+\boldsymbol{W}_{\varepsilon}^{k}, \boldsymbol{\chi}-\boldsymbol{U}_{\varepsilon}^{k}\right)^{h} \geq \gamma^{-1}\left(A^{+} \boldsymbol{U}_{\varepsilon}^{k-1}, \boldsymbol{\chi}-\boldsymbol{U}_{\varepsilon}^{k}\right)^{h}
\end{gathered}
$$

where $\boldsymbol{U}_{\varepsilon}^{0} \in \boldsymbol{K}^{h}$ is an approximation of $\mathbf{u}_{\gamma}^{0}$ as in (1.11d), e.g. $\boldsymbol{U}_{\varepsilon}^{0} \equiv \boldsymbol{\pi}^{h} \boldsymbol{u}^{0}$. Existence and (partial) uniqueness of a solution to $(2.5 \mathrm{a}, \mathrm{b})$ can be established on considering the EulerLagrange variational inequality of an appropriate strictly convex minimization problem, see [4] for details. In addition, it is easily established that the solution to $(2.5 \mathrm{a}, \mathrm{b})$ is unconditionally stable and conserves mass, i.e. it satisfies a discrete analogue of (1.12). Here, we only quote the following result. 
Lemma. 2.1 Let $\varepsilon>0, h>0$ and let $\boldsymbol{U}_{\varepsilon}^{0} \in \boldsymbol{K}^{h}$. Then, for any time partitioning $\left\{\tau_{k}\right\}_{k=1}^{K}$ there exists a solution $\left\{\boldsymbol{U}_{\varepsilon}, \boldsymbol{W}_{\varepsilon}\right\}_{k=1}^{K} \in\left[\boldsymbol{S}_{\geq 0}^{h} \times \boldsymbol{S}^{h}\right]^{K}$ to $(2.5 \mathrm{a}, \mathrm{b})$; and the solution $\left\{\boldsymbol{U}_{\varepsilon}\right\}_{k=1}^{K}$ is unique. Moreover, the solution is unconditionally stable and it holds that

$$
\mathcal{E}_{\gamma}^{h}\left(\boldsymbol{U}_{\varepsilon}^{k}\right) \leq \mathcal{E}_{\gamma}^{h}\left(\boldsymbol{U}_{\varepsilon}^{k-1}\right), \quad k=1 \rightarrow K,
$$

where $\mathcal{E}_{\gamma}^{h}\left(\boldsymbol{U}_{\varepsilon}^{k}\right):=\frac{\gamma}{2}\left(\nabla \boldsymbol{U}_{\varepsilon}^{k}, \nabla \boldsymbol{U}_{\varepsilon}^{k}\right)+\gamma^{-1}\left(\Psi\left(\boldsymbol{U}_{\varepsilon}^{k}\right), 1\right)^{h}$ is the natural discrete analogue of (1.2). Finally, it holds that $\int_{\Omega} \boldsymbol{U}_{\varepsilon}^{k} \mathrm{~d} x=\int_{\Omega} \boldsymbol{U}_{\varepsilon}^{0} \mathrm{~d} x$, and hence $\boldsymbol{U}_{\varepsilon}^{k} \in \boldsymbol{K}^{h}$, for all $k=1 \rightarrow$ $K$.

Proof. The proof in [4, Theorem 2.1] immediately carries over to the slightly different scaling in the free energy (1.2) considered here.

We note that at each time level of $(2.5 \mathrm{a}, \mathrm{b})$ a system of variational inequalities needs to be solved. The authors in [4] considered a splitting method that is based on the algorithm in [36], which can be efficiently solved on uniform partitionings of rectangular domains $\Omega \subset \mathbb{R}^{2}$. In this paper, on the other hand, we want to consider highly adaptive triangulations that utilize the fact that away from the diffuse interfaces only relatively coarse meshes are needed. Moreover, we also want to consider curved domains, such as the unit sphere; see Figure 8. To this end, we consider a multi-component version of the projected block Gauss-Seidel iterative solver introduced in [9] for the scalar CahnHilliard equation with degenerate mobility. We note that this extension is nontrivial. In particular, certain non-Euclidean projections have to be employed during the solution process; see below.

Adopting the obvious notation, the system $(2.5 \mathrm{a}, \mathrm{b})$ can be rewritten as: Find $\left\{\underline{\boldsymbol{U}}_{\varepsilon}^{k}, \underline{\boldsymbol{W}}_{\varepsilon}^{k}\right\} \in\left(\mathbb{R}_{\geq 0}^{N}\right)^{\mathcal{J}} \times\left(\mathbb{R}^{N}\right)^{\mathcal{J}}$ such that

$$
\begin{gathered}
\gamma \mathcal{M} \underline{\boldsymbol{U}}_{\varepsilon}^{k}+\tau_{k} \mathcal{L}^{k-1} \underline{\boldsymbol{W}}_{\varepsilon}^{k}=\underline{\boldsymbol{r}} \\
\gamma\left(\boldsymbol{V}-\underline{\boldsymbol{U}}_{\varepsilon}^{k}\right)^{T} \mathcal{B} \underline{\boldsymbol{U}}_{\varepsilon}^{k}-\left(\boldsymbol{V}-\underline{\boldsymbol{U}}_{\varepsilon}^{k}\right)^{T}\left(\mathcal{M} \underline{\boldsymbol{W}}_{\varepsilon}^{k}+\gamma^{-1} \mathcal{A}^{-} \underline{\boldsymbol{U}}_{\varepsilon}^{k}\right) \geq\left(\boldsymbol{V}-\underline{\boldsymbol{U}}_{\varepsilon}^{k}\right)^{T} \underline{\boldsymbol{s}} \quad \forall \boldsymbol{V} \in\left(\mathbb{R}_{\geq 0}^{N}\right)^{\mathcal{J}},
\end{gathered}
$$

where $\mathcal{M}, \mathcal{B}$ and $\mathcal{L}^{k-1}$ are symmetric $\mathcal{J} \times \mathcal{J}$ block matrices, $\mathcal{J}:=\# J$, with entries

$$
\begin{aligned}
& \mathcal{M}_{i j}:=\left(\chi_{i}, \chi_{j}\right)^{h} \operatorname{id}_{N}, \quad \mathcal{B}_{i j}:=\left(\nabla \chi_{i}, \nabla \chi_{j}\right) \operatorname{id}_{N}, \quad \mathcal{L}_{i j}^{k-1}:=\left(\nabla \chi_{i}, \nabla \chi_{j}\right) L_{\varepsilon}\left(\boldsymbol{U}_{\varepsilon}^{k-1}\right), \\
& \mathcal{A}_{i j}^{ \pm}:=\left(\chi_{i}, \chi_{j}\right)^{h} A^{ \pm}, \quad \text { and } \quad \underline{\boldsymbol{r}}:=\gamma \mathcal{M} \underline{\boldsymbol{U}}_{\varepsilon}^{k-1} \in\left(\mathbb{R}^{N}\right)^{\mathcal{J}}, \quad \underline{\boldsymbol{s}}:=\gamma^{-1} \mathcal{A}^{+} \underline{\boldsymbol{U}}_{\varepsilon}^{k-1} \in\left(\mathbb{R}^{N}\right)^{\mathcal{J}} .
\end{aligned}
$$

Let $\mathcal{L}^{k-1} \equiv \mathcal{L}_{D}-\mathcal{L}_{L}-\mathcal{L}_{L}^{T}$, with $\mathcal{L}_{L}$ and $\mathcal{L}_{D}$ being the lower (block) triangular and diagonal parts of the matrix $\mathcal{L}^{k-1}$, similarly for $\mathcal{B}$. We use this formulation in constructing our "Gauss-Seidel type" iterative method to solve $(2.5 \mathrm{a}, \mathrm{b})$.

Given $\left\{\boldsymbol{U}_{\varepsilon}^{k, 0}, \boldsymbol{W}_{\varepsilon}^{k, 0}\right\} \in \boldsymbol{S}_{\geq 0}^{h} \times \boldsymbol{S}^{h}$, for $m \geq 1$ find $\left\{\underline{\boldsymbol{U}}_{\varepsilon}^{k, m}, \underline{\boldsymbol{W}}_{\varepsilon}^{k, m}\right\} \in\left(\mathbb{R}_{\geq 0}^{N}\right)^{\mathcal{J}} \times\left(\mathbb{R}^{N}\right)^{\mathcal{J}}$ such that

$$
\begin{aligned}
& \gamma \mathcal{M} \underline{\boldsymbol{U}}_{\varepsilon}^{k, m}+\tau_{k}\left(\mathcal{L}_{D}-\mathcal{L}_{L}\right) \underline{\boldsymbol{W}}_{\varepsilon}^{k, m}=\underline{\boldsymbol{r}}+\tau_{k} \mathcal{L}_{L}^{T} \underline{\boldsymbol{W}}_{\varepsilon}^{k, m-1} \\
& \begin{aligned}
\left.\boldsymbol{V}-\underline{\boldsymbol{U}}_{\varepsilon}^{k, m}\right)^{T}\left(\gamma\left(\mathcal{B}_{D}-\mathcal{B}_{L}\right) \underline{\boldsymbol{U}}_{\varepsilon}^{k, m}-\left(\mathcal{M} \underline{\boldsymbol{W}}_{\varepsilon}^{k, m}+\gamma^{-1} \mathcal{A}^{-} \underline{\boldsymbol{U}}_{\varepsilon}^{k, m}\right)\right) \\
\geq\left(\boldsymbol{V}-\underline{\boldsymbol{U}}_{\varepsilon}^{k, m}\right)^{T}\left(\underline{\boldsymbol{s}}+\gamma \underline{\mathcal{B}}_{L}^{T} \underline{\boldsymbol{U}}_{\varepsilon}^{k, m-1}\right) \quad \forall \boldsymbol{V} \in\left(\mathbb{R}_{\geq 0}^{N}\right)^{\mathcal{J}} .
\end{aligned}
\end{aligned}
$$


We note that $(2.8 \mathrm{a}, \mathrm{b})$ can be solved explicitly for $j=1 \rightarrow \mathcal{J}$, and for each vertex $j \in J$ a system of the following type has to be solved: Find $\left\{\underline{\boldsymbol{U}}_{j}, \underline{\boldsymbol{W}}_{j}\right\} \in \mathbb{R}_{\geq 0}^{N} \times \mathbb{R}^{N}$ such that

$$
\begin{aligned}
& m_{j} \underline{\boldsymbol{U}}_{j}+L_{j j} \underline{\boldsymbol{W}}_{j}=\underline{\widehat{\boldsymbol{r}}}_{j} \\
& {\left[\boldsymbol{V}-\underline{\boldsymbol{U}}_{j}\right]^{T}\left[\left(k_{j} \mathrm{id}_{N}-\gamma^{-1} m_{j} A^{-}\right) \underline{\boldsymbol{U}}_{j}-m_{j} \underline{\boldsymbol{W}}_{j}-\underline{\widehat{\boldsymbol{s}}}_{j}\right] \geq 0 \quad \forall \boldsymbol{V} \in \mathbb{R}_{\geq 0}^{N},}
\end{aligned}
$$

where $m_{j}=\left(\chi_{j}, \chi_{j}\right)^{h}, k_{j}=\gamma\left(\nabla \chi_{j}, \nabla \chi_{j}\right), L_{j j}=\gamma^{-1} \tau_{k} \mathcal{L}_{j j}^{k-1}$. Also $\underline{\hat{\boldsymbol{r}}}=\gamma^{-1} \underline{\boldsymbol{r}}+$ $\gamma^{-1} \tau_{k}\left(\mathcal{L}_{L} \underline{\boldsymbol{W}}_{\varepsilon}^{k, m}+\mathcal{L}_{L}^{T} \underline{\boldsymbol{W}}_{\varepsilon}^{k, m-1}\right)$ and $\underline{\hat{\boldsymbol{s}}}=\underline{\boldsymbol{s}}+\gamma\left(\mathcal{B}_{L} \underline{\boldsymbol{U}}_{\varepsilon}^{k, m}+\mathcal{B}_{L}^{T} \underline{\boldsymbol{U}}_{\varepsilon}^{k, m-1}\right)$. If we assume for the moment that the matrix $L_{j j}$ is nonsingular, then $(2.9 \mathrm{a}, \mathrm{b})$ can be reformulated as: Find $\underline{\boldsymbol{U}}_{j} \in \mathbb{R}_{\geq 0}^{N}$ such that for all $\boldsymbol{V} \in \mathbb{R}_{\geq 0}^{N}$

$$
\left[\boldsymbol{V}-\underline{\boldsymbol{U}}_{j}\right]^{T} L_{j j}^{-1}[\underbrace{\left(k_{j} L_{j j}-\gamma^{-1} m_{j} L_{j j} A^{-}+m_{j}^{2} \mathrm{id}_{N}\right)}_{=: C} \underline{\boldsymbol{U}}_{j}-\underbrace{\left(m_{j} \underline{\boldsymbol{\underline { \boldsymbol { r } }}}_{j}+L_{j j} \underline{\boldsymbol{s}}_{j}\right)}_{=: \boldsymbol{g}}] \geq 0 .
$$

Clearly, the solution to $(2.10)$ is $\underline{\boldsymbol{U}}_{j}=P_{\geq 0}\left(C^{-1} \boldsymbol{g}\right)$, where $P_{\geq 0}$ is the orthogonal projection onto the convex set $\mathbb{R}_{\geq 0}^{N}$ with respect to the weighted inner product $\langle x, y\rangle_{\star}:=x^{T} L_{j j}^{-1} C y$. However, we recall from (2.3) that $L_{j j} \mathbf{1}=\mathbf{0}$, and so we cannot simply invert equation (2.9a). On restricting $L_{j j}$ to be a map $L_{j j}: \mathcal{H}^{N} \rightarrow \mathcal{H}^{N}$, recall (1.10), one can introduce an appropriate inverse and make (2.10) rigorous. Alternatively, one can introduce the nonsingular matrix $\widetilde{L}_{j j}:=L_{j j}+\omega \mathbf{1} \mathbf{1}^{T}$, where $\omega:=\max _{n, p=1 \rightarrow N}\left|\left\{L_{j j}\right\}_{n p}\right|$, which turns out to be more convenient in practice. Then it is easy to see that (2.9a) can be equivalently written as

$$
m_{j} \underline{\boldsymbol{U}}_{j}+\widetilde{L}_{j j} \underline{\boldsymbol{W}}_{j}=\underline{\widehat{\boldsymbol{r}}}_{j}+\beta \mathbf{1}, \quad \mathbf{1}^{T} \underline{\boldsymbol{U}}_{j}=1,
$$

where $\beta \in \mathbb{R}$ is unknown. Then, similarly to (2.10), we define

$$
\widetilde{C}:=k_{j} \widetilde{L}_{j j}-\gamma^{-1} m_{j} \widetilde{L}_{j j} A^{-}+m_{j}^{2} \operatorname{id}_{N}
$$

and $\widetilde{\boldsymbol{g}}:=m_{j} \underline{\widehat{\boldsymbol{r}}}_{j}+\widetilde{L}_{j j} \underline{\widehat{\boldsymbol{s}}}_{j}$, so that we need to solve the system: Find $\underline{\boldsymbol{U}}_{j} \in \mathbb{R}_{\geq 0}^{N}$ and $\beta \in \mathbb{R}$ such that $\mathbf{1}^{T} \underline{\boldsymbol{U}}_{j}=1$, i.e. $\underline{\boldsymbol{U}}_{j} \in \mathbb{Q}^{N}$, and

$$
\left[\boldsymbol{V}-\underline{\boldsymbol{U}}_{j}\right]^{T} \widetilde{L}_{j j}^{-1}\left[\widetilde{\boldsymbol{C}} \underline{\boldsymbol{U}}_{j}-\left(\widetilde{\boldsymbol{g}}+m_{j} \beta \mathbf{1}\right)\right] \geq 0 \quad \forall \boldsymbol{V} \in \mathbb{R}_{\geq 0}^{N} .
$$

Choosing $\boldsymbol{V}$ with $\mathbf{1}^{T} \boldsymbol{V}=1$ in (2.13), on noting that $\widetilde{L}_{j j}^{-1} \mathbf{1}=\frac{1}{\omega N} \mathbf{1}$, yields that $\underline{\boldsymbol{U}}_{j} \in \mathbb{Q}^{N}$ satisfies the variational inequality

$$
\left[\boldsymbol{V}-\underline{\boldsymbol{U}}_{j}\right]^{T} \widetilde{L}_{j j}^{-1}\left[\widetilde{C} \underline{\boldsymbol{U}}_{j}-\widetilde{\boldsymbol{g}}\right] \geq 0 \quad \forall \boldsymbol{V} \in \mathbb{Q}^{N} .
$$

Hence the solution of $(2.14)$, similarly to $(2.10)$, is given by

$$
\underline{\boldsymbol{U}}_{j}=\widetilde{P}_{\mathbb{Q}^{N}}\left(\widetilde{C}^{-1} \widetilde{\boldsymbol{g}}\right),
$$

where $\widetilde{P}_{\mathbb{Q}^{N}}$ is the orthogonal projection onto $\mathbb{Q}^{N}$ with respect to the weighted inner product $\langle x, y\rangle_{\widetilde{\star}}:=x^{T} \widetilde{L}_{j j}^{-1} \widetilde{C} y=x^{T}\left(k_{j} \operatorname{id}_{N}-\gamma^{-1} m_{j} A^{-}+m_{j}^{2} \widetilde{L}_{j j}^{-1}\right) y$. Observe that $\widetilde{L}_{j j}^{-1} \widetilde{C}$ is clearly symmetric positive definite. Having obtained $\underline{\boldsymbol{U}}_{j}$, we can compute $\underline{\boldsymbol{W}}_{j}$ as follows. 
Let $n \in\{1, \ldots, N\}$ be such that $\underline{U}_{j}^{n}>0$. Then choosing $\boldsymbol{V}=\underline{\boldsymbol{U}}_{j} \pm \frac{1}{2} \underline{U}_{j}^{n} \boldsymbol{e}_{n}$ in (2.13), it follows that

$$
\boldsymbol{e}_{n}^{T} \widetilde{L}_{j j}^{-1}\left[\widetilde{C} \underline{\boldsymbol{U}}_{j}-\left(\widetilde{\boldsymbol{g}}+m_{j} \beta \mathbf{1}\right)\right]=0
$$

and hence

$$
\beta=\frac{\boldsymbol{e}_{n}^{T} \widetilde{L}_{j j}^{-1}\left[\widetilde{C} \underline{\boldsymbol{U}}_{j}-\widetilde{\boldsymbol{g}}\right]}{m_{j} \boldsymbol{e}_{n}^{T} \widetilde{L}_{j j}^{-1} \mathbf{1}}=\omega N m_{j}^{-1}\left\langle\boldsymbol{e}_{n}, \underline{\boldsymbol{U}}_{j}-\widetilde{C}^{-1} \widetilde{\boldsymbol{g}}\right\rangle_{\widetilde{\star}} .
$$

Finally, the solution to $(2.9 \mathrm{a}, \mathrm{b})$ is completed by

$$
\underline{\boldsymbol{W}}_{j}=\widetilde{L}_{j j}^{-1}\left(\underline{\underline{\boldsymbol{r}}}_{j}+\beta \mathbf{1}-m_{j} \underline{\boldsymbol{U}}_{j}\right) .
$$

In order to solve $(2.15)$, and hence $(2.9 \mathrm{a}, \mathrm{b})$, it remains to find $\widetilde{P}_{\mathbb{Q}^{N}}(\boldsymbol{x})$ for an arbitrary $\boldsymbol{x} \in \mathbb{R}^{N}$. Here we use the fact that $\widetilde{P}_{\mathbb{Q}^{N}}(\boldsymbol{x})=\widetilde{P}_{\mathbb{Q}^{N}}\left(\widetilde{P}_{\mathcal{H}^{N}}(\boldsymbol{x})\right)$, i.e. we first project $\boldsymbol{x}$ onto the Gibbs plane $\mathcal{H}^{N}$, and then project the result onto $\mathbb{Q}^{N}$. As $\mathcal{H}^{N}$ is an affine subspace, the former projection is straightforward. In order to derive an efficient algorithm for computing the projection $\widetilde{P}_{\mathbb{Q}^{N}}(\boldsymbol{x})$, for $\boldsymbol{x} \in \mathcal{H}^{N}$, we need the following lemma.

Lemma. 2.2 Let $\partial_{n} \mathbb{Q}^{N}:=\left\{\boldsymbol{\zeta} \in \mathbb{Q}^{N}: \zeta^{n}=0\right\} \subset \mathcal{H}_{n}^{N}:=\left\{\boldsymbol{\zeta} \in \mathcal{H}^{N}: \zeta^{n}=0\right\}$ be the face of the Gibbs simplex opposite the vertex $\boldsymbol{e}_{n}, n=1 \rightarrow N$. Let $\boldsymbol{x} \in \mathcal{H}^{N}$ and set $d_{\mathbb{Q}^{N}}(\boldsymbol{x})=\left|\widetilde{P}_{\mathbb{Q}^{N}}(\boldsymbol{x})-\boldsymbol{x}\right|_{\nwarrow}$, where $|\boldsymbol{\zeta}|_{\widetilde{\star}}:=\left[\langle\boldsymbol{\zeta}, \boldsymbol{\zeta}\rangle_{\widetilde{\star}}\right]^{\frac{1}{2}}$. Then the following hold:

(a). $d_{\mathbb{Q}^{N}}(\boldsymbol{x})=\min \left\{|\boldsymbol{\zeta}-\boldsymbol{x}|_{\widetilde{\star}}: \boldsymbol{\zeta} \in \mathbb{Q}^{N}\right\}$.

(b). If $\boldsymbol{x} \notin \mathbb{Q}^{N}$, then $d_{\mathbb{Q}^{N}}(\boldsymbol{x})=\min _{n=1 \rightarrow N} \min \left\{|\boldsymbol{\zeta}-\boldsymbol{x}|_{\widetilde{\star}}: \boldsymbol{\zeta} \in \partial_{n} \mathbb{Q}^{N}\right\}$.

(c). If $\boldsymbol{x} \notin \mathbb{Q}^{N}$ and $x^{p} \geq 0$, then $d_{\mathbb{Q}^{N}}(\boldsymbol{x})=\min _{n \neq p} \min \left\{|\boldsymbol{\zeta}-\boldsymbol{x}|_{\nwarrow}: \boldsymbol{\zeta} \in \partial_{n} \mathbb{Q}^{N}\right\}$.

(d). $\widetilde{P}_{\partial_{n} \mathbb{Q}^{N}}(\boldsymbol{x})=\widetilde{P}_{\partial_{n} \mathbb{Q}^{N}}\left(\widetilde{P}_{\mathcal{H}_{n}^{N}}(\boldsymbol{x})\right)$, where $\widetilde{P}_{A}$ is the $\widetilde{\star}$-orthogonal projection onto $A \subset \mathcal{H}^{N}$.

Proof. (a) follows immediately from the definition of $\widetilde{P}_{\mathbb{Q}^{N}}(\boldsymbol{x})$. (b) follows from $\partial \mathbb{Q}^{N}=$ $\cup_{n=1}^{N} \partial_{n} \mathbb{Q}^{N}$. (c) can be shown with the following geometric argument. Assume that $\boldsymbol{y}=\widetilde{P}_{\mathbb{Q}^{N}}(\boldsymbol{x}) \in \partial_{p} \mathbb{Q}^{N}$. We know that there exists an $N^{-} \geq 1$ such that $x^{q_{i}}<0, i=$ $1 \rightarrow N^{-}$. For all $i$ it follows, since $y^{q_{i}} \geq 0$, that the segment $[\boldsymbol{x}, \boldsymbol{y}] \subset \mathbb{R}^{N}$ intersects the hyperplane $\mathcal{H}_{q_{i}}^{N}$ in a point $\boldsymbol{z}_{i}$. Let $\boldsymbol{z}_{q_{j}}$ be the point furthest away from $\boldsymbol{x}$, i.e. $\left|\boldsymbol{z}_{q_{j}}-\boldsymbol{x}\right|_{\tilde{\star}}=$ $\max _{i=1 \rightarrow N^{-}}\left|\boldsymbol{z}_{q_{i}}-\boldsymbol{x}\right|_{\nwarrow}$. Then we have that $\boldsymbol{z}_{q_{j}} \in \partial_{q_{j}} \mathbb{Q}^{N}$ and $\left|\boldsymbol{z}_{q_{j}}-\boldsymbol{x}\right|_{\nwarrow} \leq|\boldsymbol{y}-\boldsymbol{x}|_{\tilde{\star}}$. On recalling that $q_{j} \neq p$, this implies the desired result. Finally, $(d)$ follows directly from the definitions of $\widetilde{P}_{\partial_{n} \mathbb{Q}^{N}}$ and $\widetilde{P}_{\mathcal{H}_{n}^{N}}$, and the fact that $\partial_{n} \mathbb{Q}^{N} \subset \mathcal{H}_{n}^{N}$.

We are now in a position to describe an algorithm for finding the projection $\boldsymbol{y}=$ $\widetilde{P}_{\mathbb{Q}^{N}}(\boldsymbol{x})$, where we may assume that $\boldsymbol{x} \in \mathcal{H}^{N}$. Clearly, $\boldsymbol{y}=\boldsymbol{x}$ if $\boldsymbol{x} \in \mathbb{Q}^{N}$. So from now on we assume in addition that $\boldsymbol{x} \notin \mathbb{Q}^{N}$.

If $N=2$, the projection simplifies to the standard projection onto the Gibbs simplex $\mathbb{Q}^{2}$, that is $\boldsymbol{y}=\boldsymbol{e}_{n}$, if $x^{n}>1$. 
For $N \geq 3$ we use the following routine. Let $\left\{n: x^{n}<0\right\} \equiv\left\{n_{i}: i=1 \rightarrow N^{-}\right\}$ and $\boldsymbol{y}_{n_{i}}=\widetilde{P}_{\partial_{n_{i}} \mathbb{Q}^{N}}(\boldsymbol{x}), i=1 \rightarrow N^{-}$. Then choose $\boldsymbol{y}=\boldsymbol{y}_{n_{0}}$ such that $\left|\boldsymbol{y}_{n_{0}}-\boldsymbol{x}\right|_{\tilde{\star}}=$ $\min _{i=1 \rightarrow N^{-}}\left|\boldsymbol{y}_{n_{i}}-\boldsymbol{x}\right|_{\tilde{\star}}$. On recalling Lemma 2.2(d), we compute the projection $\boldsymbol{y}_{n_{i}}=$ $\widetilde{P}_{\partial_{n_{i}} \mathbb{Q}^{N}}(\boldsymbol{x})$ by first projecting $\boldsymbol{x}$ onto the hyperplane $\mathcal{H}_{n_{i}}^{N}$ followed by a projection onto $\partial_{n_{i}} \mathbb{Q}^{N} \subset \mathcal{H}_{n_{i}}^{N}$. Here we note that as $\partial_{n_{i}} \mathbb{Q}^{N}$ is isomorphic to $\mathbb{Q}^{N-1}$, the second projection can be implemented recursively. Finally, the projection $\widetilde{P}_{\mathcal{H}_{n_{i}}^{N}}$ can be obtained as follows. Let $\mathcal{H}_{n_{i}}^{N}=\boldsymbol{e}_{p}+\operatorname{span}\left\{\boldsymbol{v}_{j}: j=1 \rightarrow N-2\right\}$, where e.g. $\boldsymbol{v}_{j}=\boldsymbol{e}_{p_{j}}-\boldsymbol{e}_{p}$ with $p_{j} \neq p \neq n_{i}$ and $p_{j} \neq n_{i}$. Define the Gram matrix $G \in \mathbb{R}^{(N-2) \times(N-2)}$ and right hand side $b \in \mathbb{R}^{N-2}$ such that

$$
G_{i j}=\left\langle\boldsymbol{v}_{i}, \boldsymbol{v}_{j}\right\rangle_{\widetilde{\star}}, \quad b_{j}=\left\langle\boldsymbol{x}-\boldsymbol{e}_{p}, \boldsymbol{v}_{j}\right\rangle_{\widetilde{\star}} \quad \forall i, j=1 \rightarrow N-2, \quad \text { and let } \quad \omega:=G^{-1} b .
$$

Then $\widetilde{P}_{\mathcal{H}_{n_{i}}^{N}}(\boldsymbol{x})=\boldsymbol{e}_{p}+\sum_{j=1}^{N-2} \omega_{i} \boldsymbol{v}_{j}$.

We note that in practice the linear systems (2.15) and (2.16), (2.17) are solved with an $L U$ and a Cholesky factorization method, respectively.

\section{Numerical results}

In this section we report on several numerical simulations for the approximation $(2.5 \mathrm{a}, \mathrm{b})$ of $\left(\mathrm{P}_{\gamma}\right)$. We will use a uniform time step size $\tau_{k}=\tau, k=1 \rightarrow K$ and set $\varepsilon=10^{-5}$ throughout. Unless otherwise stated, the domain is given by the unit cube $\Omega=\left(-\frac{1}{2}, \frac{1}{2}\right)^{d}$. In addition, for $N \neq 3$ we let $A$ be given by (1.4), so that $A^{-}=-\frac{N-1}{N} \mathbf{1} \mathbf{1}^{T}$. If $N=3$, on the other hand, we let $A$ be defined by

$$
A=-\left(\begin{array}{ccc}
0 & 1 & \alpha \\
1 & 0 & 1 \\
\alpha & 1 & 0
\end{array}\right)
$$

where $\alpha \in \mathbb{R}_{>0}$. We note that now $A^{-}=-\frac{1}{s}\left(\begin{array}{ccc}\frac{(\alpha+s)^{2}}{8} & \frac{\alpha+s}{2} & \frac{(\alpha+s)^{2}}{8} \\ \frac{\alpha+s}{2} & 2 & \frac{\alpha+s}{2} \\ \frac{(\alpha+s)^{2}}{8} & \frac{\alpha+s}{2} & \frac{(\alpha+s)^{2}}{8}\end{array}\right)$, where $s:=\left[\alpha^{2}+8\right]^{\frac{1}{2}}$. Unless otherwise stated, we always choose $\alpha=1$.

For the iterative algorithm (2.8a,b) we set, for $k \geq 1,\left\{\boldsymbol{U}_{\varepsilon}^{k, 0}, \boldsymbol{W}_{\varepsilon}^{k, 0}\right\} \equiv\left\{\boldsymbol{U}_{\varepsilon}^{k-1}, \boldsymbol{W}_{\varepsilon}^{k-1}\right\}$, where $\boldsymbol{U}_{\varepsilon}^{0}=\boldsymbol{\pi}^{h} \mathbf{u}_{\gamma}^{0}$ and $\boldsymbol{W}_{\varepsilon}^{0} \in \boldsymbol{S}^{h}$ is defined, on recalling $(2.6 \mathrm{~b})$, by $\underline{\boldsymbol{W}}_{\varepsilon}^{0}=\gamma \mathcal{M}^{-1} \mathcal{B} \underline{\boldsymbol{U}}_{\varepsilon}^{0}-$ $\gamma^{-1}\left(\mathcal{A}^{-}+\mathcal{A}^{+}\right) \underline{\boldsymbol{U}_{\varepsilon}^{0}}$, and adopted the stopping criterion

$$
\left|\underline{\boldsymbol{U}}_{\varepsilon}^{k, m}-\underline{\boldsymbol{U}}_{\varepsilon}^{k, m-1}\right|_{\infty}<t o l,
$$

with tol $=10^{-7}$, where $|\underline{\boldsymbol{V}}|_{\infty}:=\max _{j=1 \rightarrow \mathcal{J}}\left|\underline{\boldsymbol{V}}_{j}\right|$ for $\underline{\boldsymbol{V}} \in\left(\mathbb{R}^{N}\right)^{\mathcal{J}}$. Once the stopping criterion is satisfied, we set $\left\{\boldsymbol{U}_{\varepsilon}^{k}, \boldsymbol{W}_{\varepsilon}^{k}\right\} \equiv\left\{\boldsymbol{U}_{\varepsilon}^{k, m}, \boldsymbol{W}_{\varepsilon}^{k, m}\right\}$.

For ease of notation, we introduce the definition

$$
\boldsymbol{U}_{\varepsilon}(t):=\frac{1}{\tau}\left(\left(t-t_{k-1}\right) \boldsymbol{U}_{\varepsilon}^{k}+\left(t_{k}-t\right) \boldsymbol{U}_{\varepsilon}^{k-1}\right) \quad t \in\left[t_{k-1}, t_{k}\right] \quad k \geq 1 .
$$


In order to define the initial shape of the bubbles we introduce the following function. Given $z \in \mathbb{R}^{d}, a \in \mathbb{R}^{d}$ with $\min \left\{a_{1}, a_{2}, a_{3}\right\}=1$ and $R \in \mathbb{R}_{>0}$ we define

$$
v(z, a, R ; x):= \begin{cases}1 & r(z, a ; x)-R \leq-\frac{\gamma \pi}{2} \\ \frac{1}{2}-\frac{1}{2} \sin \left(\frac{r(z, a ; x)-R}{\gamma}\right) & |r(z, a ; x)-R|<\frac{\gamma \pi}{2} \\ 0 & r(z, a ; x)-R \geq \frac{\gamma \pi}{2}\end{cases}
$$

where $(r(z, a ; x)-R)$ denotes a suitable distance function for e.g. an ellipsoid centred around $z$ with semi axis $a_{1} R, \ldots, a_{d} R$ or a torus with radius $a_{1}$ and tubular radius $R$. E.g. $r(z, a ; x) \equiv r_{e}(z ; x):=\left[\sum_{i=1}^{d}\left(\frac{x_{i}-z_{i}}{a_{i}}\right)^{2}\right]^{\frac{1}{2}}$ for an ellipse and $r(z, a ; x) \equiv r_{t}(z, a ; x):=$ $\left[\left(a_{1}-\left[\sum_{i=1}^{d-1}\left(x_{i}-z_{i}\right)^{2}\right]^{\frac{1}{2}}\right)^{2}+\left(x_{d}-z_{d}\right)^{2}\right]^{\frac{1}{2}}$ for a torus. In line with the asymptotics of the phase field approach, see Section 1, the interfacial thickness for $v$ is not less than $\gamma \pi$. We note that for the choice (3.1), the thickness of the interface between phases 1 and 3 is asymptotically given by $\frac{\gamma \pi}{\sqrt{\alpha}}$. Hence, for $\alpha \neq 1$, we adapt the profile (3.4) for the function $v_{3}$ in (3.5), below, accordingly. For the initial data $\mathbf{u}_{\gamma}^{0}$ to $\left(\mathrm{P}_{\gamma}\right)$, we choose a sequence of functions $v_{1}, \ldots, v_{N}$, where $v_{1} \equiv 1$ and $v_{n}, n=2 \rightarrow N$, are of the form (3.4), and then set

$$
\left\{\mathbf{u}_{\gamma}^{0}\right\}^{n}(x)=v_{n}(x) \prod_{p=n+1}^{N}\left(1-v_{p}(x)\right) .
$$

It is easy to see that the following algorithm produces the initial values (3.5). For $p=$ $1 \rightarrow N$ set $y_{p}^{p}(x):=v_{p}(x)$, and let $y_{n}^{p}(x):=y_{n}^{p-1}(x)\left(1-v_{p}(x)\right)$ for $n=1 \rightarrow p-1$. Finally, set $\mathbf{u}_{\gamma}^{0}(x):=\left(y_{1}^{N}(x), \ldots, y_{N}^{N}(x)\right)^{T} \in \mathbb{Q}^{N}$, where the latter claim follows inductively from the given construction.

For the graphical plots in this section we will use the zero contour sets of the function $p_{1}\left(\boldsymbol{U}_{\varepsilon}\right)$ to visualize the bubble boundaries, where

$$
p_{n}(\boldsymbol{U})=\max _{i=1 \rightarrow N}\left\{\left|\boldsymbol{U}-\boldsymbol{e}_{n}\right|^{2}-\left|\boldsymbol{U}-\boldsymbol{e}_{i}\right|^{2}\right\}, \quad n=1 \rightarrow N
$$

In addition, for $d=2$ we will give the zero contour lines of the functions $p_{n}\left(\boldsymbol{U}_{\varepsilon}\right), n=2 \rightarrow$ $N-1$, wherever $p_{1}\left(\boldsymbol{U}_{\varepsilon}\right)>0$. For the plots in $\mathbb{R}^{3}$ we used the package GRAPE (Graphics Programming Environment).

\subsection{Adaptive mesh}

The finite element approximation $(2.5 \mathrm{a}, \mathrm{b})$ was implemented in the framework of the adaptive finite element toolbox Alberta 1.2, see [41]. In particular, we utilized the possibility to employ highly adaptive finite element meshes and implemented a mesh refinement strategy that is based on the two dimensional approximation for the scalar Cahn-Hilliard equation in [9], see also [3] for $d=3$. Here we make use of the fact that the evolution will concentrate inside the interfacial regions $\left\{\boldsymbol{U}_{\varepsilon}^{k-1} \neq \boldsymbol{e}_{n}, n=1 \rightarrow N\right\}$. Hence we want to use a refined mesh in these interfacial regions, and a coarser mesh away from the interfaces, inside the pure phases $\left\{\boldsymbol{U}_{\varepsilon}^{k-1}=\boldsymbol{e}_{n}\right\}, n=1 \rightarrow N$. 
We now describe our mesh refinement strategy. Here we assume that $\bar{\Omega} \subset\left[-\frac{1}{2}, \frac{1}{2}\right]^{d}$. We choose two integer parameters $N_{f}>N_{c}$ and set $h_{f}=\frac{1}{N_{f}}, h_{c}=\frac{1}{N_{c}}$. Then we set

$$
v o l_{f}=\frac{h_{f}^{d}}{d !} \quad \text { and } \quad v_{c}=\frac{h_{c}^{d}}{d !}
$$

that is, for $d=3, v o l_{f}$ denotes the volume of a tetrahedron with three right-angled and isosceles faces with side length $h_{f}$, while for $d=2$ it denotes the area of a right-angled and isosceles triangle with side length $h_{f}$, and similarly for $v_{o l}$.

We start with an initial triangulation $\mathcal{T}^{0}$ consisting of uniform elements $\sigma$ for which $|\sigma| \leq v o l_{f}$, and fix the two parameters $\delta_{f}=t o l \times 10^{-1}$ and $\delta_{c}=t o l \times 10^{-3}$, where tol is the prescribed tolerance from (3.2). Then, for $k \geq 1$ given $\boldsymbol{U}_{\varepsilon}^{k-1}$ and a partition $\mathcal{T}^{k-1}$, an element is marked for refinement if it satisfies

$$
\delta_{f}<\eta_{\sigma}:=1-\max _{n=1 \rightarrow N} \frac{1}{|\sigma|} \int_{\sigma}\left\{U_{\varepsilon}^{k-1}\right\}^{n}(x) \mathrm{d} x .
$$

Note that the indicator $\eta_{\sigma}$ in (3.7) is zero if and only if $\boldsymbol{U}_{\varepsilon}^{k-1} \equiv \boldsymbol{e}_{n}$ on $\sigma$ for an $n \in$ $\{1, \ldots, N\}$, i.e. when $\sigma$ lies entirely inside one of the pure phases. If a marked element's volume satisfies $|\sigma| \geq 2 \mathrm{vol}_{f}$, it is refined into two smaller elements via bisectioning of its longest edge. An element is marked for coarsening if it satisfies $|\sigma| \leq \frac{1}{2} \mathrm{vol}_{c}$ and $\eta_{\sigma}<\delta_{c}$. An element that is marked for coarsening is coarsened only if all its neighbouring elements are marked for coarsening as well. Once all the elements have been visited and a refinement or coarsening indication has been assigned as discussed above, then the mesh is updated accordingly. This marking and refinement process is repeated until no more elements have been refined or coarsened. Thus we obtain the triangulation $\mathcal{T}^{k}$ on which the new solution $\boldsymbol{U}_{\varepsilon}^{k}$ will be computed. We note that in practice only at the first time step $k=1$, more then 1 of the described refinement cycles are needed.

Finally, throughout the numerical simulations in this section we choose $N_{f}$ such that there are always at least approximately 6 mesh points across each interface in each direction. In addition, unless otherwise stated, we choose $N_{c}=2$ throughout.

\subsection{Numerical results for $d=2$}

\subsubsection{Double bubbles}

We conducted the following convergence experiments for the evolution of two elliptical bubbles that merge to form a standard double bubble, i.e. $N=3$, with the two bubbles and the surrounding fluid each represented by one component. Recall that the standard double bubble is given by two arcs of a circle with a straight line in between them, see e.g. [31]. The initial setup for our experiments is given by two $2: 3$ ellipses with semi minor axis 0.15 and centres $( \pm 0.18,0)^{T} \in \mathbb{R}^{2}$. We repeated the same experiment with decreasing values of $\gamma$, i.e. $\gamma=\frac{1}{6 \pi}, \frac{1}{12 \pi}, \frac{1}{24 \pi}$. In particular, we set $T=0.01, \tau=72(\gamma \pi)^{2} \times 10^{-5}$ 

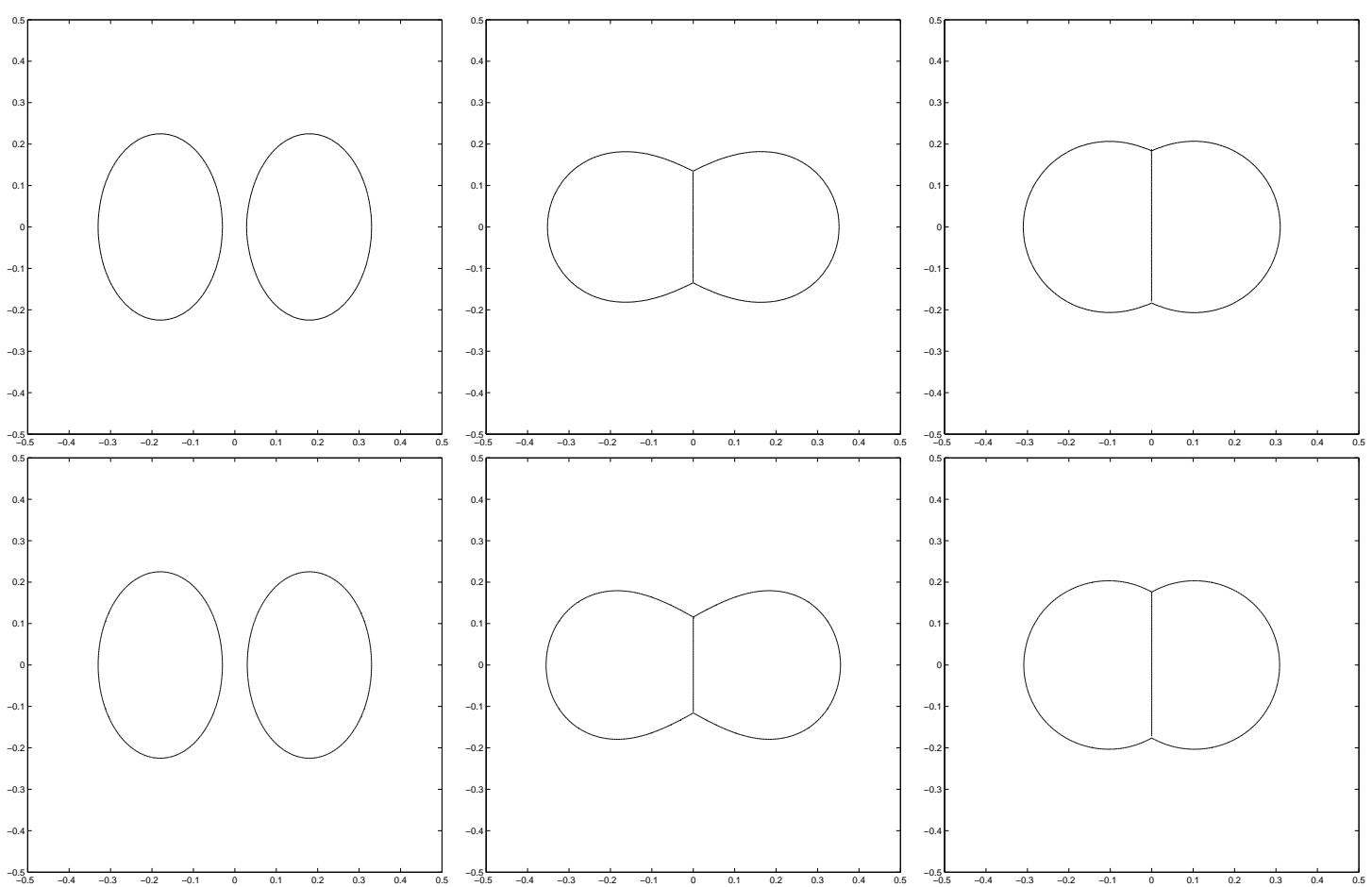

Figure 1: The solution $\boldsymbol{U}_{\varepsilon}(t)$ visualized at times $t=0,5 \times 10^{-4}, T=0.01$ for $\gamma=\frac{1}{6 \pi}$ and $\gamma=\frac{1}{24 \pi}$ (from top to bottom).

and used the appropriate refinement parameters $N_{f}=\frac{32}{3} \frac{1}{\gamma \pi}$ and $N_{c}=\frac{N_{f}}{8}$. See Figure 1, where we plot the results for $\gamma=\frac{1}{6 \pi}$ and $\gamma=\frac{1}{24 \pi}$, by visualizing the appropriate zero contour lines of the functions defined in (3.6). We omitted the results for $\gamma=\frac{1}{12 \pi}$, as they look very similar. In addition, for the computation for $\gamma=\frac{1}{24 \pi}$ we show the adaptive mesh at times $t=0$ and $t=T$ as well as a comparison between the computed solution and the true sharp interface solution in Figure 2. Here we use the fact that the steady state solution for this flow is the exact standard double bubble shape, where the involved radii can be computed from the given volume fractions as described in the Appendix. We note that the two solutions are graphically indistinguishable and hence we are satisfied that our numerical method can accurately predict energy minimizing constellations of soap bubble clusters. We stress that this is an improvement on the approach in [47], where e.g. the solution shown in [47, Fig. 6] clearly differs from the standard double bubble solution.

We now compare the previous evolution to an example, where the surface energy densities are not equal, so that the triple junction angles will deviate from $120^{\circ}$. In particular, the observed angles are predicted by Young's Law

$$
\frac{\sin \phi_{1}}{\varsigma_{23}}=\frac{\sin \phi_{2}}{\varsigma_{13}}=\frac{\sin \phi_{3}}{\varsigma_{12}} ;
$$

where $\varsigma_{i j}$ is the energy density of the interface between the pure phases $\boldsymbol{e}_{i}$ and $\boldsymbol{e}_{j}$, recall (1.13), and $\phi_{n}$ is the angle inside phase $n$ at a triple junction, opposite the $(i, j)$-interface, where $i, j \neq n$. It follows from (3.1) that $\varsigma_{12}=\varsigma_{23}$, and so (3.8) implies that the angles at a triple junction are given by $\left(\pi-\frac{\phi_{2}}{2}, \phi_{2}, \pi-\frac{\phi_{2}}{2}\right)$ with $\phi_{2}=2 \arccos \left(\frac{1}{2} \frac{\varsigma_{13}}{\varsigma_{12}}\right)$. We choose 

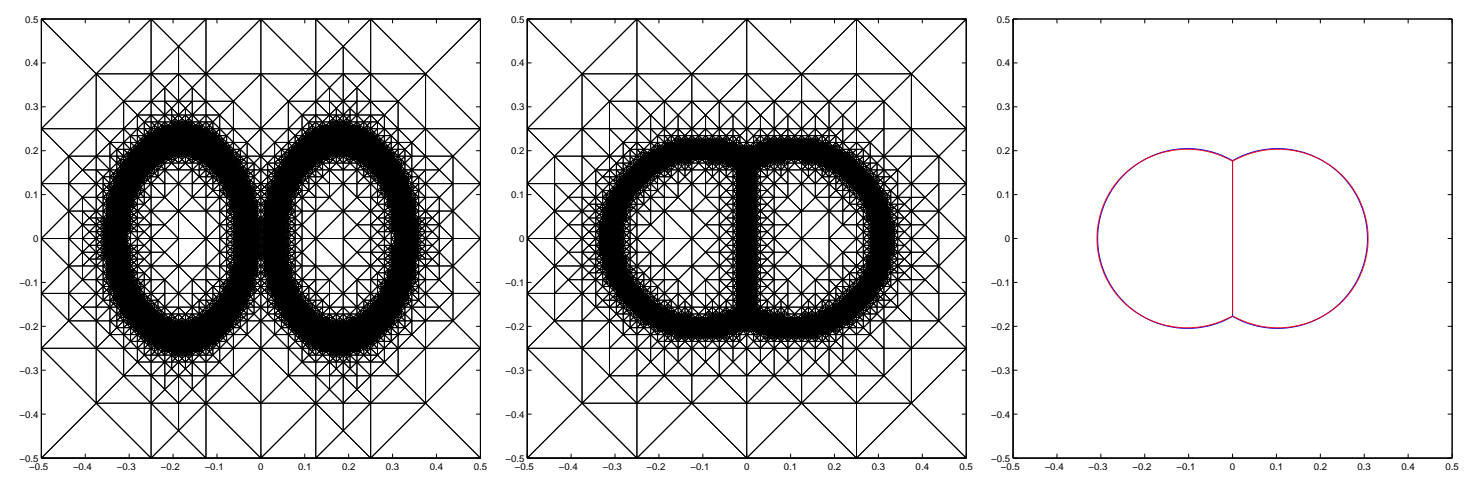

Figure 2: $\left(\gamma=\frac{1}{24 \pi}\right)$ Adaptive mesh at times $t=0, T=0.01$ and a comparison between the computed solution $\boldsymbol{U}_{\varepsilon}(T)$ (red) and the exact sharp interface solution (blue), which are graphically indistinguishable.

$\alpha \in\left\{\frac{1}{4}, \frac{4}{5}, \frac{5}{4}, 2\right\}$ in (3.1) and start the evolution with the same parameters as in Figure 1 for $\gamma=\frac{1}{12 \pi}$. The results can be seen in Figure 3. We note that as the energy of the right most interface is now larger(smaller), its length gets penalized more(less) and consequently in the steady state configuration it is shorter(longer) than in Figure 1. We also want to compare the four computed equal area steady state solutions with the exact energy minimizer. To this end, we determine the two surface energies $\varsigma_{13}$ and $\varsigma_{12}$ numerically, similarly to the technique used in [7]. That is, for the two pairs $(i, j)=(1,2)$ and $(1,3)$ we split the domain $\Omega$ into two pure phases $\boldsymbol{e}_{i}, \boldsymbol{e}_{j}$, with a vertically aligned straight interface between them. Using this setup, we compute the evolution of the corresponding Allan-Cahn version of the scheme $(2.5 \mathrm{a}, \mathrm{b})$ until a steady state has been reached. This resulting standing wave will then approximate the energy minimizing profile in (1.13), and hence provides a numerical value for the energy density $\varsigma_{i j}$. We note that if one can guarantee that the standing wave minimizing profile in (1.13) stays on the boundary $\partial \mathbb{Q}^{N}$ of the Gibbs simplex, then one can compute the energy density $\varsigma_{i j}$ exactly, and the expected angle is then given by $\frac{\varsigma_{13}}{\varsigma_{12}}=\sqrt{\alpha}$. We stress that for the numerical estimation of the densities $\varsigma_{i j}$, it is crucial to replace the Cahn-Hilliard approximation (2.5a,b) with an analogous Allan-Cahn approximation, as otherwise the mass conservation property of (2.5a,b) (recall Lemma 2.1) prevents any other phase from being present in the standing wave profile, which in general would lead to an overestimation of $\varsigma_{i j}$.

The numerically determined surface energy densities for the different values of $\alpha$ lead to the following angles at the triple junctions: $\phi_{2}=150.9^{\circ}, 126.8^{\circ}, 112^{\circ}$ and $90.4^{\circ}$, respectively. We compare the computed steady state solutions to the exact double bubble shapes for these triple junction angles, and the comparisons can be seen in Figure 4. We note that there is excellent agreement between our phase field solutions and the true sharp interface energy minimizers. Once again we refer to the Appendix for the details on how to compute the true sharp interface solutions.

Next we provide an example for an evolution for two bubbles with nonequal area. The initial data is given by two superimposed circles of radius 0.25 and 0.15 with centres at $(-0.15,0)^{T}$ and $(0.1,0)^{T}$, respectively. The results are shown in Figure 5 , where we 

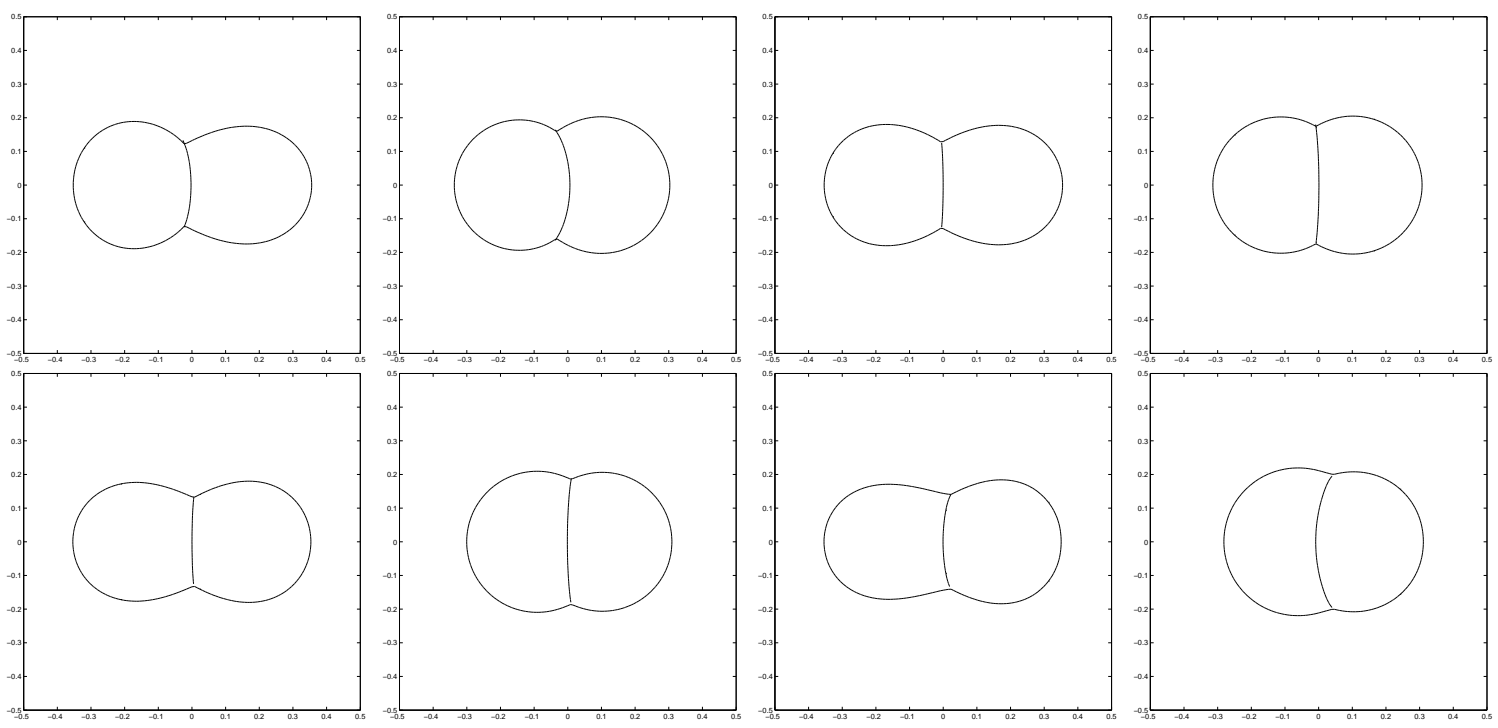

Figure 3: $\left(\gamma=\frac{1}{12 \pi}\right)$ Equal area double bubble for different surface energy densities. The solution $\boldsymbol{U}_{\varepsilon}(t)$ is shown at times $t=5 \times 10^{-4}, T=0.01$ for $\alpha=\frac{1}{4}, \frac{4}{5}, \frac{5}{4}$ and 2 (from top left to bottom right).
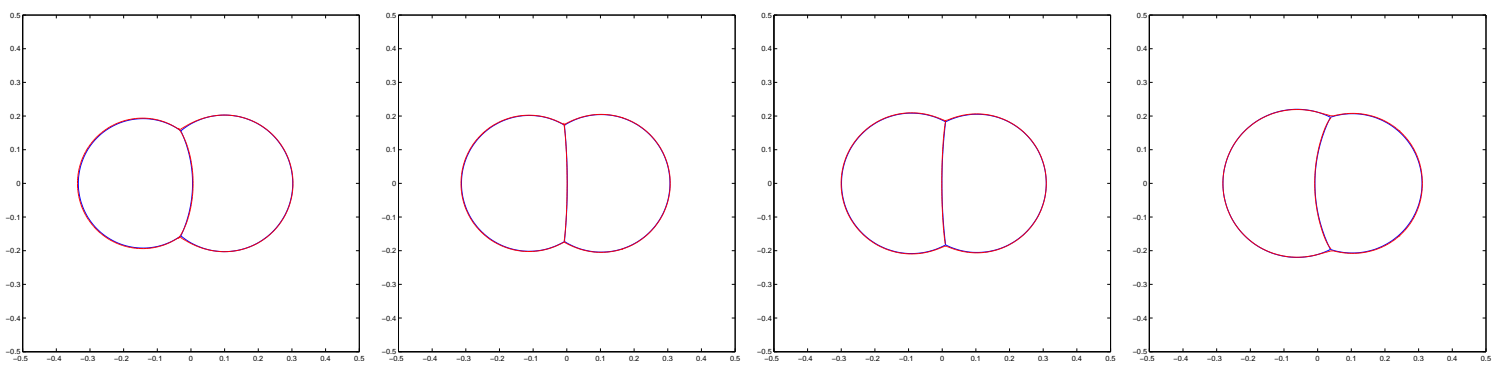

Figure 4: $\left(\gamma=\frac{1}{12 \pi}\right)$ Comparison between the computed solution $\boldsymbol{U}_{\varepsilon}(T)$ (red) and the exact sharp interface solution (blue) for $\alpha=\frac{1}{4}, \frac{4}{5}, \frac{5}{4}$ and 2 .

used the same discretization parameters as in Figure 1 for $\gamma=\frac{1}{12 \pi}$. Once again we note the excellent agreement between the computed numerical steady state and the true sharp interface solution.

\subsubsection{Triple bubbles}

In the next experiment we investigate the evolution of three equal area bubbles towards the standard triple bubble, so here $N=4$. The initial bubbles are given by two elliptical bubbles as in Figure 1, now centred at $( \pm 0.18,-0.22)^{T}$, plus a rotated bubble of the same type centred at $(-0.18,0.22)^{T}$. The interfacial parameter is $\gamma=\frac{1}{12 \pi}$ and the chosen discretization parameters are $N_{f}=128, \tau=10^{-6}$ and $T=0.05$. The evolution can be seen in Figure 6, where we note once more that our method can easily deal with topological changes. Moreover, the numerical steady state solution shows the standard triple bubble for three equal area bubbles, i.e. three straight line segments meeting at a triple junction 

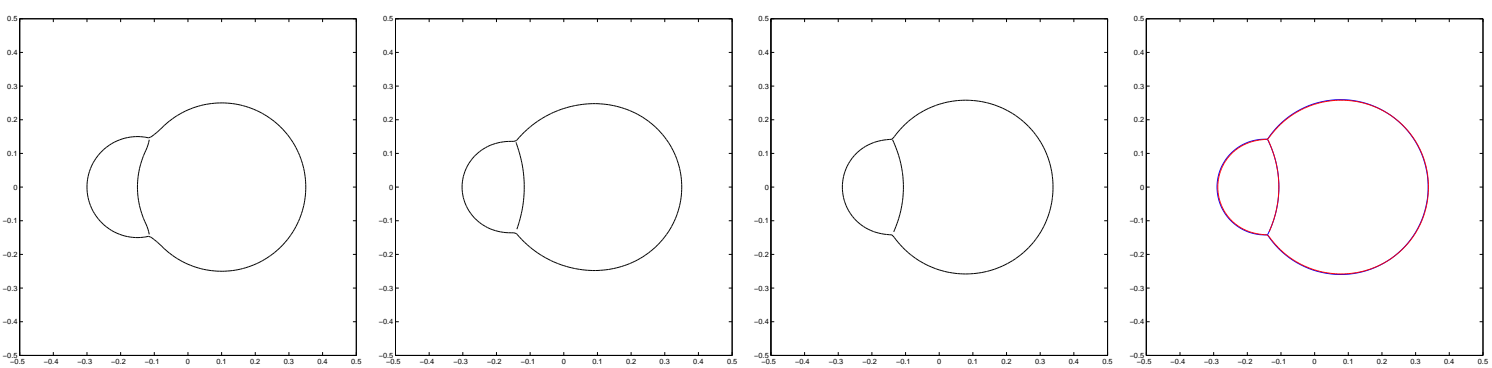

Figure 5: $\left(\gamma=\frac{1}{12 \pi}\right)$ A nonequal area double bubble. The solution $\boldsymbol{U}_{\varepsilon}(t)$ is shown at times $t=0,5 \times 10^{-4}, T=0.01$. A comparison of $\boldsymbol{U}_{\varepsilon}(T)$ (red) to the exact solution (blue) is shown on the right.
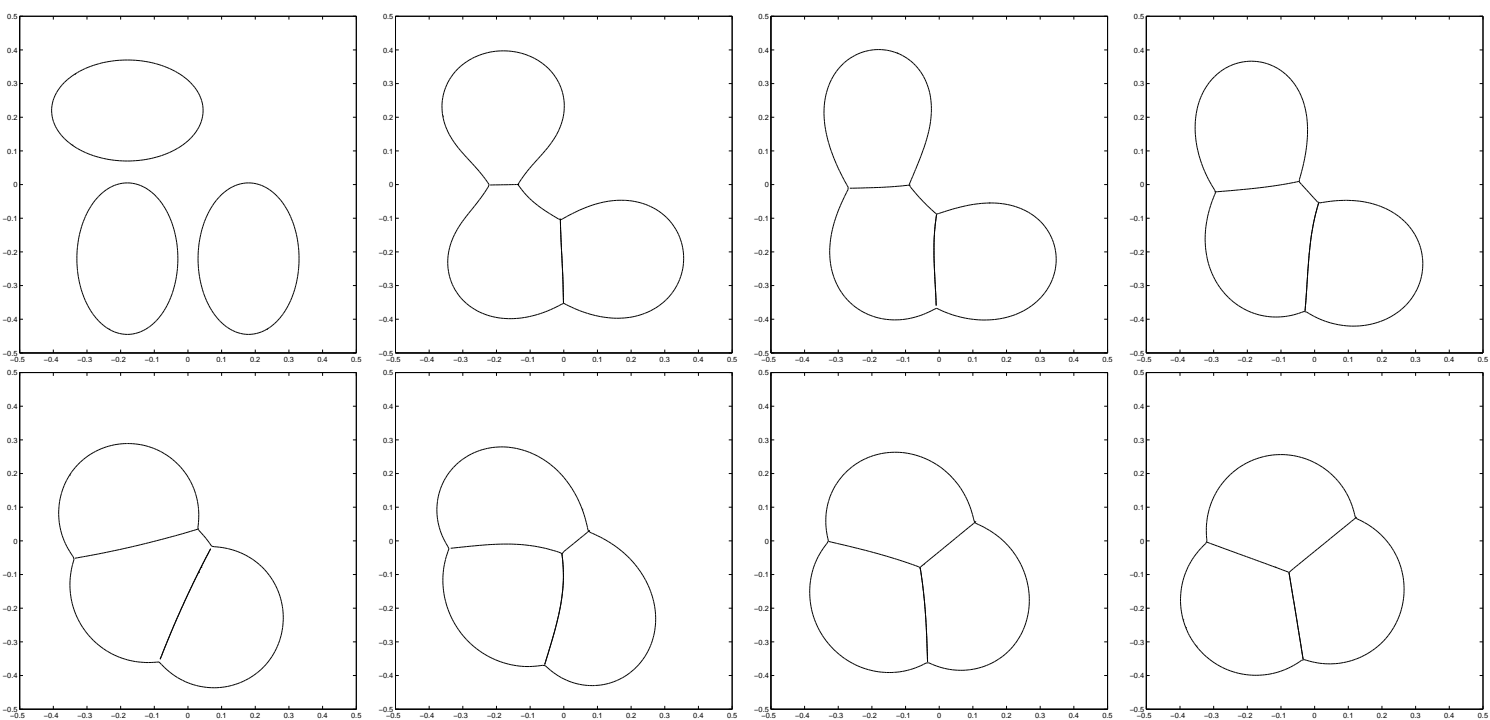

Figure 6: $\left(\gamma=\frac{1}{12 \pi}\right)$ The solution $\boldsymbol{U}_{\varepsilon}(t)$ at times $t=0,5 \times 10^{-4}, 0.001,0.002,0.01$, $0.015,0.02, T=0.05$.

and three circle segments, which in pairs meet the straight line segments at three further triple junctions. All the triple junction angles are equal to $120^{\circ}$. As previously stated, the proof that this configuration is indeed the surface energy minimizer among all the curve networks enclosing and separating three equal volumes was given only very recently in [46].

\subsubsection{Boundary intersections}

In this subsection, we consider the surface diffusion flow for curves and networks of curves that intersect the external boundary $\partial \Omega$. The first example is for a nonconvex domain $\Omega$, see Figure 7. The boundary of the domain $\Omega$ is given by the parameterization $r: S^{1} \rightarrow \mathbb{R}^{2}$, where $r(z)=\left(\frac{3}{8}+\frac{1}{8} \cos (4 \theta)\right) z$. We show two different evolutions for this domain. One is for an initial circle of radius 0.2 centred at $(0.2,0.15)^{T}$, and the second is for the same circle now centred at $(0.1,0.15)^{T}$. The discretization parameters are $N_{f}=128, \tau=10^{-5}$, 

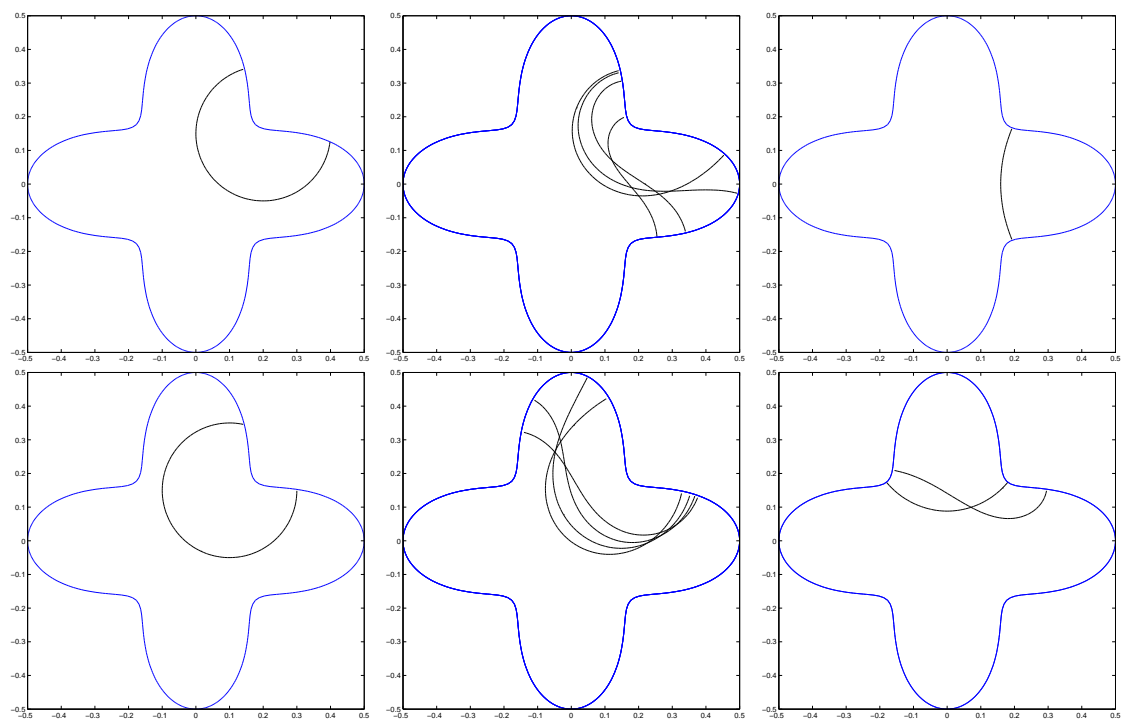

Figure 7: $\left(\gamma=\frac{1}{12 \pi}\right)$ The solution $\boldsymbol{U}_{\varepsilon}(t)$ at times $t=0, t=0.002,0.005,0.007,0.008$ and $t=0.01,0.05$.

$T=0.05$. In both cases one can observe that the interface moves along the boundary $\partial \Omega$ until it has reached a profile that partitions the domain according to the initially given area fractions by a curve of shortest length. We note that similar numerical experiments for a direct approximation of surface diffusion for curves have been conducted in [5], while theoretical aspects on the stability of configurations as in Figure 7 have been considered in $[24]$.

Next we study the evolution of curve networks inside the unit disk, so that the steady state solutions are candidates for the least perimeter partitions of the disk as considered in [14]. We note that there only a proof for the well known least perimeter partition of the disk into three regions of prescribed area is given, whereas for the case $N \geq 4$ only conjectures exist so far. We start with the case $N=3$ and show the evolution of two different triple junctions inside the circular domain $\Omega:=\left\{x \in \mathbb{R}^{2}:|x|<\frac{1}{2}\right\}$ in Figure 8. The first experiment is for two superimposed circles with radius 0.3 and 0.4 , centred at $(0.3,0.25)^{T}$ and $(0.25,-0.25)^{T}$, respectively. The initial setup for the second experiment consists of a circle of radius 0.3 centred at $(0.3,-0.1)^{T}$ that is joined by a straight horizontal line through $(0,-0.1)^{T}$. The discretization parameters for both experiments are $N_{f}=128, \tau=5 \times 10^{-6}$ and $T=0.05$. Some examples for the case of $N=4$ present phases are shown in Figure 9 . The initial setups are given by (a) two circles of radius 0.2 centred at $( \pm 0.3,-0.1)^{T}$ that are connected by a straight horizontal line through $(0,-0.1)^{T}$ and (b) by three superimposed circles of radii $0.4,0.3$ and 0.3 centred at $(0.25,-0.25)^{T},(0.3,0.25)^{T}$ and $(0,-0.25)^{T}$, respectively. The discretization parameters are as before. Finally, we show a partition for $N=5$ and $N=6$ in Figure 10. The initial setups are given by (a) three superimposed circles of radii $0.3,0.2$ and 0.2 centred at $(0,-0.1)^{T}$ and $( \pm 0.3, \pm 0.25)^{T}$, respectively, and (b) the same three circles with an additional vertical line through $(-0.3,0)^{T}$. The discretization parameters are chosen as before. 

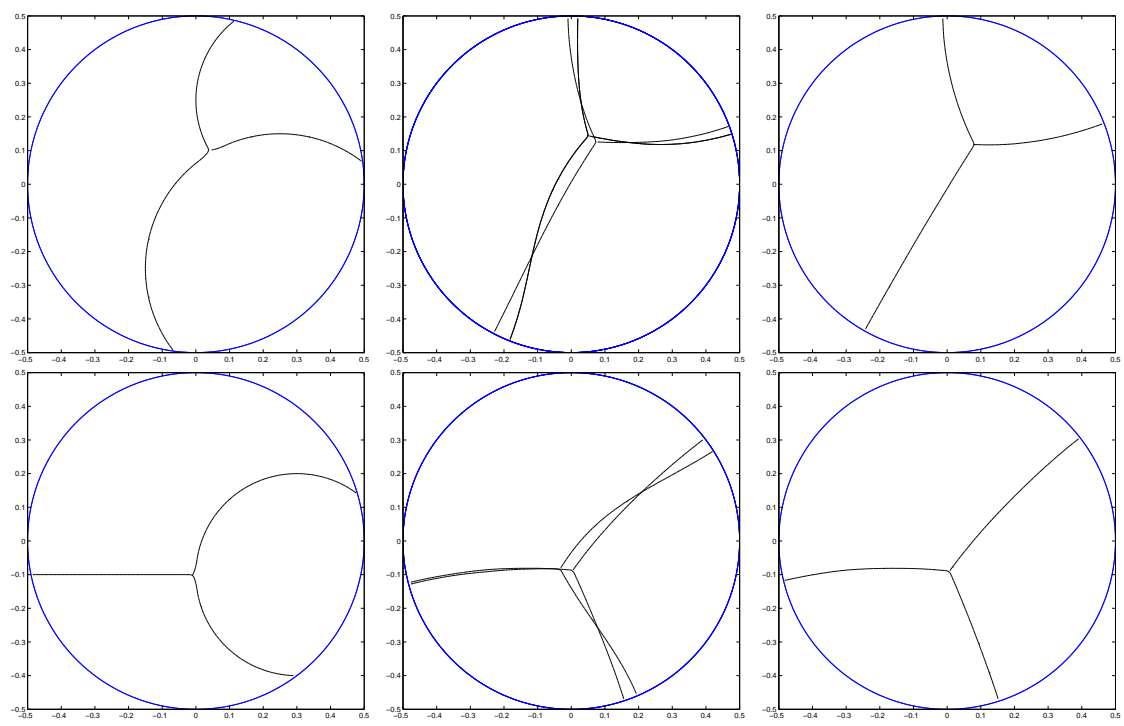

Figure 8: $\left(\gamma=\frac{1}{12 \pi}\right)$ Evolution of two triple junctions inside the a disk. The solution $\boldsymbol{U}_{\varepsilon}(t)$ at times $t=0,0.002,0.01,0.05$.
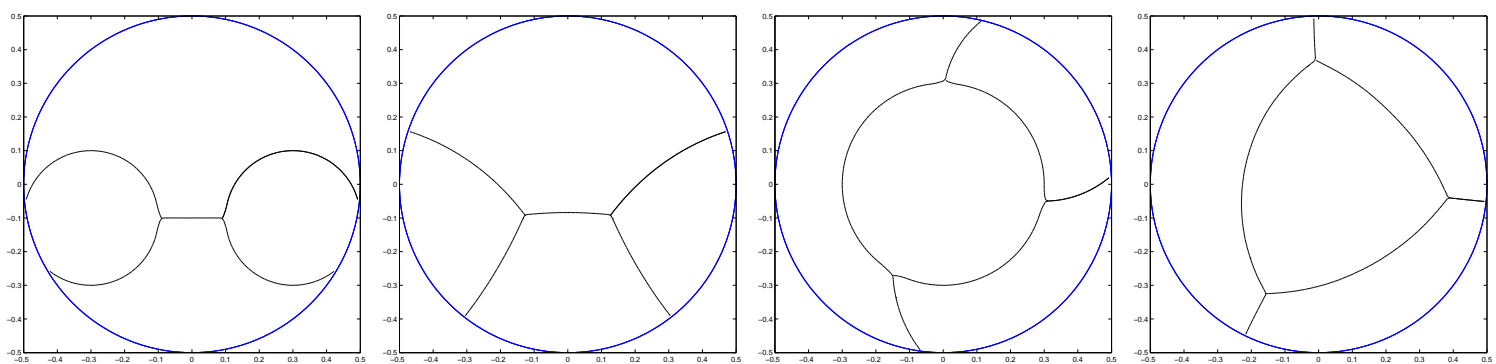

Figure 9: $\left(\gamma=\frac{1}{12 \pi}\right)$ Two evolutions for a four phase partition of a disk. The solution $\boldsymbol{U}_{\varepsilon}(t)$ at times $t=0,0.05$.
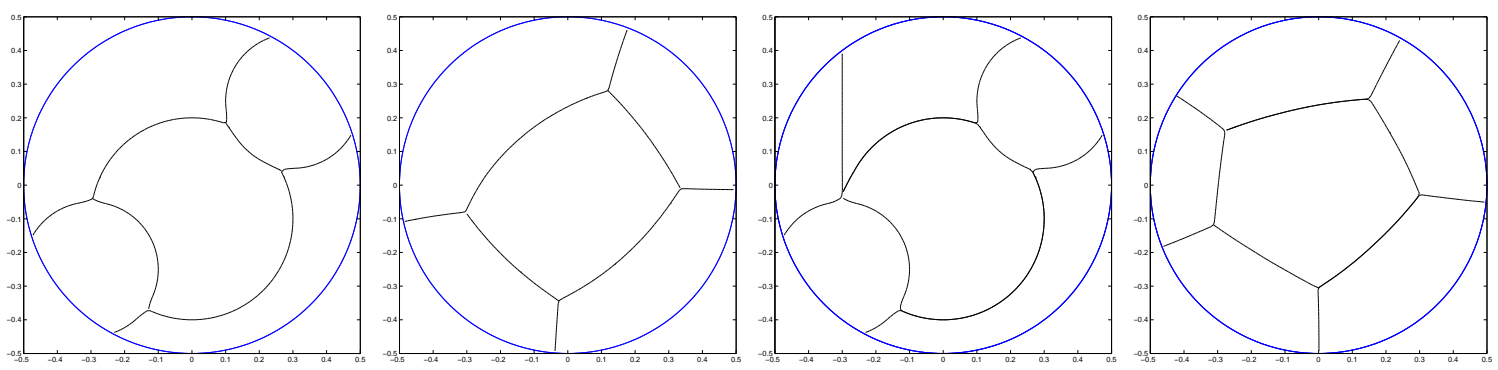

Figure 10: $\left(\gamma=\frac{1}{12 \pi}\right)$ Evolutions for a five and a six phase partition of a disk. The solution $\boldsymbol{U}_{\varepsilon}(t)$ at times $t=0,0.05$. 

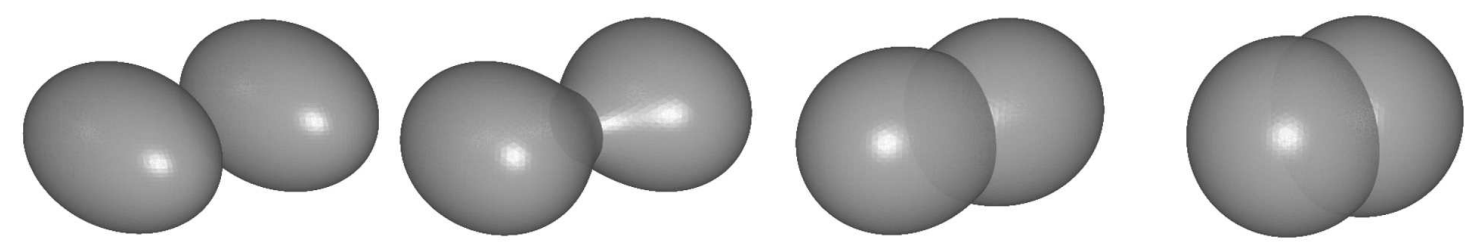

Figure 11: $\left(\gamma=\frac{1}{12 \pi}\right)$ Evolution towards the symmetric standard double bubble in 3d. Solution at times $t=0,10^{-4}, 10^{-3}, 8 \times 10^{-3}$.

We note that in each of our simulations, the numerical solutions attain a steady state that corresponds to the conjectured least perimeter partition of the unit disk for the given $N$ areas. That is, each interface (or curve segment) has constant mean curvature, i.e. is a straight line or a circle segment, and intersects the external boundary at an angle of $90^{\circ}$ or meets two other interfaces at a triple junction with an angle of $120^{\circ}$.

\subsection{Numerical results for $d=3$}

\subsubsection{Standard double bubbles}

In our first numerical simulation in three space dimensions we investigate the evolution of two ellipsoidal bubbles to the standard double bubble. In the equal volume case the standard bubble is given by two symmetric arches of two sphere segments that touch a flat disc between them at an angle of $120^{\circ}$. The evolution towards this standard double bubble is shown in Figure 11. We note that the evolution undergoes a change of topology when the two bubbles merge, and that this poses no difficulties for the employed phase field approximation. The chosen discretization parameters are $N_{f}=128, \tau=5 \times 10^{-7}$, $T=0.008$. The initial setup is given by two $2: 3: 2$ ellipsoids with semi minor axis 0.15 and centres $( \pm 0.18,0,0)^{T} \in \mathbb{R}^{3}$. In addition, we also show an evolution for a non-equal volume standard double bubble. To this end, we start with two superimposed spheres, where the larger one has radius 0.25 and is centred around $(0.1,0,0)^{T}$, while the smaller one has radius 0.15 and is centred around $(-0.15,0,0)^{T}$. The evolution of this setup to the standard double bubble can be seen in Figure 12. The discretization parameters are chosen as before.

\subsubsection{Nonstandard double bubbles}

Here we investigate numerically the stability of so-called nonstandard double bubbles, where one bubble wraps around the other. Such bubble configurations are also called torus bubbles, see e.g. [29, Fig. 7] for an illustration. Until the double bubble conjecture was proven, these configurations were candidates for stable surface energy minimizing 

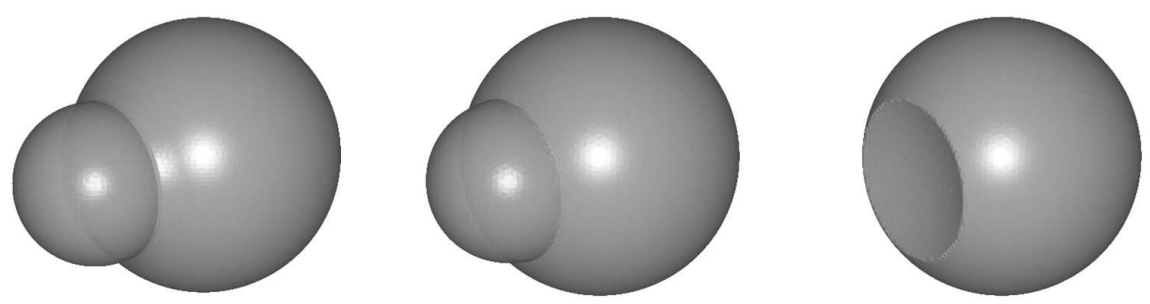

Figure 12: $\left(\gamma=\frac{1}{12 \pi}\right)$ A nonsymmetric standard double bubble in 3d. Solution at times $t=0, T=8 \times 10^{-3}$, and the second bubble at time $T$.
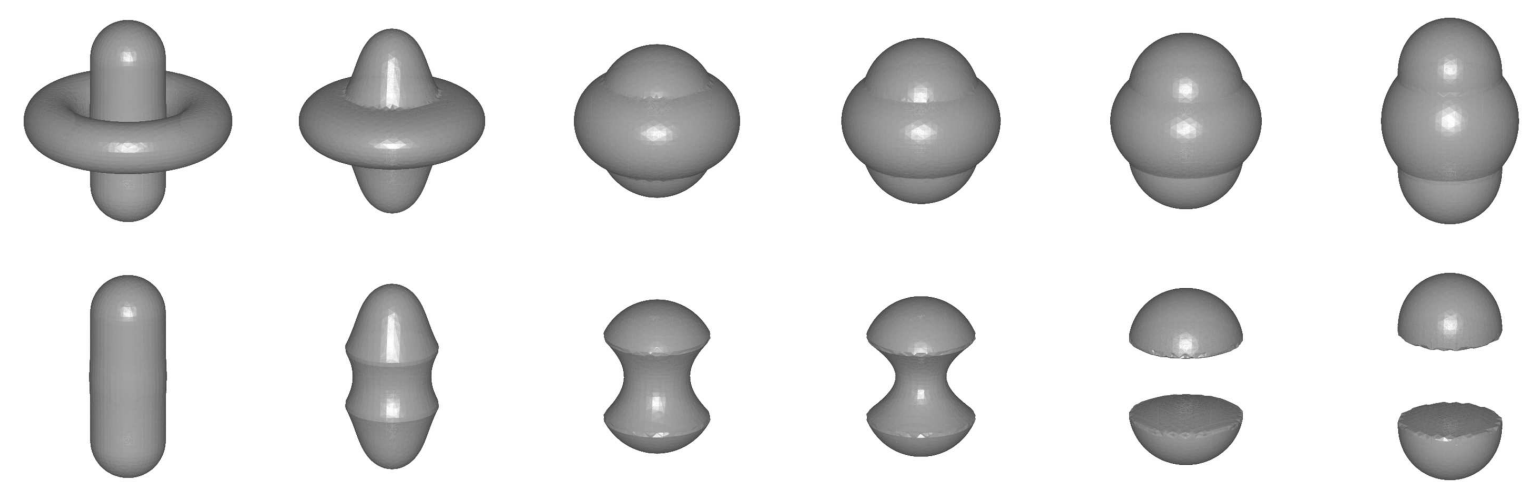

Figure 13: $\left(\gamma=\frac{1}{6 \pi}\right)$ Evolution of nonstandard double bubble with a thin torus. Solution at times $t=0,10^{-4}, 10^{-3}, 2.5 \times 10^{-3}, 3 \times 10^{-3}, 0.05$. Below we show the evolution of the cylindric part of the double bubble.

double bubbles, and so there is great interest in their modelling. However, along with their recent proof of the double bubble conjecture in [31], the authors also showed that the nonstandard double bubble configuration is unstable. This is confirmed by our numerical simulations, see e.g. Figure 14. We note that the method in [47] apparently failed to correctly simulate the evolution of a torus bubble. E.g. in [47, Fig. 7] it appears that a torus bubble is shown as a local energy minimizer. In contrast to that, we are satisfied that the results in Figures 13 and 14 are accurate simulations of the evolution of torus bubbles.

For our simulation in Figure 13 we started with an initial setup of two nontouching bubbles, where one is a torus with radius 0.27 and tubular radius 0.09 , and the other is a "cigar like" shape, i.e. a union of a cylinder and two half spheres, of total dimensions $0.23 \times 0.23 \times 0.728$. The discretization parameters are $N_{f}=64, \tau=5 \times 10^{-6}$ and $T=0.05$.

Next we compute the evolution of a similar torus bubble, where the torus part of the bubble is thinner than before and is no longer aligned at the middle of the cylindric bubble. Again a change in topology occurs when the torus part of the bubble merges to a ball. But this time the initially cylindric bubble does not split up into two parts. Instead, 

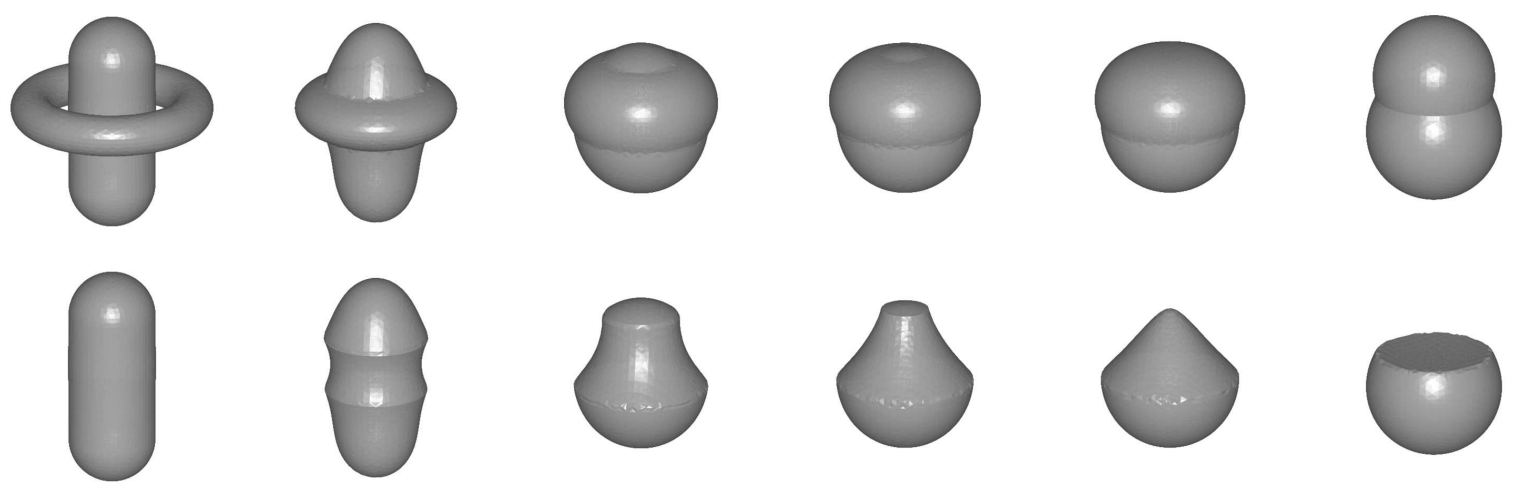

Figure 14: $\left(\gamma=\frac{1}{6 \pi}\right)$ Evolution of a nonstandard double bubble with a thin torus. Solution at times $t=0,10^{-4}, 10^{-3}, 0.018,0.02,0.05$. Below we show the evolution of the cylindric part of the double bubble.

a standard double bubble forms with both components occupying connected regions in space. The numerical results can be seen in Figure 14. We used the same discretization parameters as before. The initial setup is given by a torus with radius 0.28 and tubular radius 0.07 , together with a cigar like shape of total dimensions $0.3 \times 0.3 \times 0.75$. The corresponding evolution of the interface for the second bubble is shown in the lower half of Figure 14.

\subsubsection{Triple bubbles}

Finally, we present a simulation for a triple bubble in three space dimensions. The initial setup consists of three $3: 2: 2$ ellipsoids with semiminor axis 0.15 and centred at $(-0.14,0,-0.19)^{T},(0.22,0,-0.19)^{T}$ and $(-0.14,0,0.24)^{T}$, respectively. The discretization parameters are $N_{f}=64, \tau=5 \times 10^{-6}$ and $T=0.05$. The results can be seen in Figure 15 . We note that the numerical steady state exhibits three flat surfaces, together with three spherical ones. We also note that there are two quadruple junction points, where in each case four triple junction lines meet. To our knowledge, these are the first numerical results for the evolution of triple bubbles in $\mathbb{R}^{3}$ in the literature.

\section{Conclusion}

We have presented a phase field (diffuse interface) model for the evolution of immiscible fluid clusters. The introduced finite element approximation together with the employed adaptive mesh strategy allows for accurate and efficient numerical simulations of these clusters. To this end, a novel iterative solution method involving nontrivial projections to the Gibbs simplex was introduced. The accuracy of the numerical method was tested with the help of exact solutions for $d=2$, and several numerical experiments for $d=2$ and $d=3$ were presented, for up to $N=6$ different fluids. The presented numerical results 

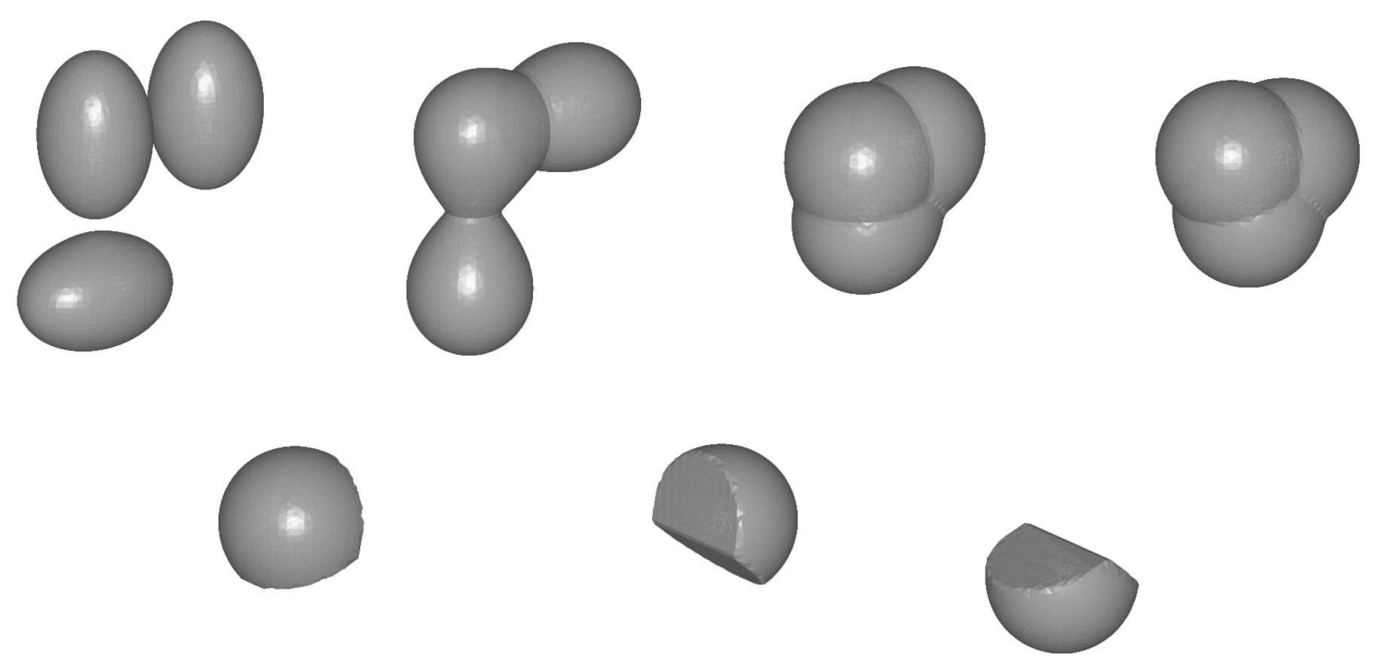

Figure 15: $\left(\gamma=\frac{1}{6 \pi}\right)$ A symmetric triple bubble in 3d. Solution at times $t=0,5 \times 10^{-4}, 5 \times$ $10^{-3}, T=0.05$. Below the three separate bubbles making up the triple bubble at time $T$.

will be of particular interest to researchers in materials science, chemical engineering as well as in geometric measure theory.

\section{A Appendix}

Here we discuss how to construct the exact double bubble solutions used in Figures 2, 4 and 5 . Let $d=2$ and let the two enclosed areas $v_{1}, v_{2}$ as well as the triple junction angles $\left(\phi_{1}, \phi_{2}, \phi_{3}\right)$ be given. We need to find three circular arcs of the form

$$
\Gamma_{i}:=\left\{\left(\rho_{i}+r_{i} \cos \theta, r_{i} \sin \theta\right): \theta \in I_{i}\right\}, \quad i=1 \rightarrow 3,
$$

where

$$
I_{1}:=\left[\theta_{1}, 2 \pi-\theta_{1}\right], \quad I_{2}:=\left[\pi-\theta_{2}, \pi+\theta_{2}\right], \quad I_{3}:=\left[-\theta_{3}, \theta_{3}\right],
$$

and where $\rho_{i}, r_{i}$ and $\theta_{i}$ are unknown, see Figure 16. Here we have assumed that the double bubble is aligned with the $x_{1}$-axis and that the two triple junction points lie on the $x_{2}$-axis. At first, for simplicity, we assume that $\rho_{3}<0$ so that the centre of the circle segment $\Gamma_{3}$ lies in the left half plane.

It is now our aim to derive a set of nonlinear equations for the unknowns $\rho_{i}, r_{i}, \theta_{i}$, $i=1 \rightarrow 3$. It turns out that the nine unknowns can be expressed in terms of $r_{1}, r_{2}$, $\theta_{3}$ and the data $\left(\phi_{1}, \phi_{2}, \phi_{3}\right)$ alone. First, a simple geometric argument yields that e.g. $\theta_{1}-\theta_{3}=\pi-\phi_{2}$ and hence

$$
\theta_{1}=\pi-\phi_{2}+\theta_{3} \quad \text { and similarly } \quad \theta_{2}=\pi-\phi_{1}-\theta_{3} .
$$




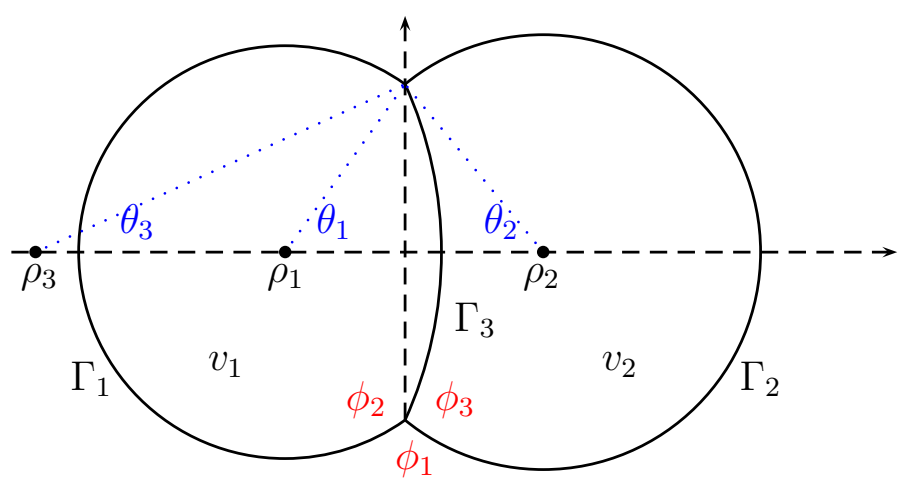

Figure 16: An example double bubble for $v_{1}=v_{2}$ and $\phi_{2}=150^{\circ}$.

Second, it obviously holds that

$$
\rho_{i}=(-1)^{i} r_{i} \cos \theta_{i}, \quad i=1 \rightarrow 3 .
$$

Finally, we can express $r_{3}$ in terms of $r_{1}$ and $r_{2}$. To see this, recall from Young's law (3.8) that we can express the ratios of surface energies as

$$
\frac{\varsigma_{13}}{\varsigma_{12}}=\frac{\sin \phi_{2}}{\sin \phi_{3}} \quad \text { and } \quad \frac{\varsigma_{23}}{\varsigma_{12}}=\frac{\sin \phi_{1}}{\sin \phi_{3}} .
$$

Moreover, it follows from the balance of flux condition

$$
\varsigma_{23} \frac{1}{r_{1}}-\varsigma_{13} \frac{1}{r_{2}}-\varsigma_{12} \frac{1}{r_{3}}=0
$$

see e.g. [27], that

$$
r_{3}=\left[\frac{\varsigma_{23}}{\varsigma_{12}} \frac{1}{r_{1}}-\frac{\varsigma_{13}}{\varsigma_{12}} \frac{1}{r_{2}}\right]^{-1} .
$$

The relationship (A.4) for the equal energy case $\varsigma_{12}=\varsigma_{23}=\varsigma_{13}$ was stated in e.g. [32].

Combining (A.1)-(A.4) yields that there are really only three unknowns: $r_{1}, r_{2}$ and $\theta_{3}$. Hence it is sufficient to prescribe three independent equations. This is now straightforward on recalling the area formula for a circular segment:

$$
a_{i}:=r_{i}^{2}\left(\theta_{i}-\sin \theta_{i} \cos \theta_{i}\right), \quad i=1 \rightarrow 3,
$$

where e.g. $a_{3}$ is the area enclosed by $\Gamma_{3}$ and the $x_{2}$-axis. Then three possible equations that need to hold are

$$
\begin{aligned}
v_{1} & =\pi r_{1}^{2}-a_{1}+a_{3}, \\
v_{2} & =\pi r_{2}^{2}-a_{2}-a_{3}, \\
0 & =r_{1} \sin \theta_{1}-r_{2} \sin \theta_{2} .
\end{aligned}
$$

The equations (A.6a-c) can be solved with a nonlinear optimization method. In practice, a Nelder-Mead simplex method, [40], with the starting value $\left(r_{1}, r_{2}, \theta_{3}\right)=\left(\left(\frac{v_{1}}{\pi}\right)^{\frac{1}{2}},\left(\frac{v_{2}}{\pi}\right)^{\frac{1}{2}}, 0\right)$ always converged.

Finally, the case $\rho_{3}>0$ can be treated in an analogous fashion and one finds once again that the equations $(\mathrm{A} .6 \mathrm{a}-\mathrm{c})$ need to hold. 


\section{References}

[1] F. Almgren, J. E. Taylor, Soap bubble clusters, Forma 11 (3) (1996) 199-207.

[2] S. Baldo, Minimal interface criterion for phase transitions in mixtures of CahnHilliard fluids, Ann. Inst. H. Poincaré Anal. Non Linéaire 7 (2) (1990) 67-90.

[3] L'. Baňas, R. Nürnberg, Finite element approximation of a three dimensional phase field model for void electromigration, J. Sci. Comp.(to appear).

[4] J. W. Barrett, J. F. Blowey, H. Garcke, On fully practical finite element approximations of degenerate Cahn-Hilliard systems, M2AN Math. Model. Numer. Anal. 35 (2001) 713-748.

[5] J. W. Barrett, H. Garcke, R. Nürnberg, On the variational approximation of combined second and fourth order geometric evolution equations, SIAM J. Sci. Comput. 29 (3) (2007) 1006-1041.

[6] J. W. Barrett, H. Garcke, R. Nürnberg, A parametric finite element method for fourth order geometric evolution equations, J. Comput. Phys. 222 (1) (2007) 441-467.

[7] J. W. Barrett, H. Garcke, R. Nürnberg, A phase field model for the electromigration of intergranular voids, Interfaces Free Bound. 9 (2) (2007) 171-210.

[8] J. W. Barrett, H. Garcke, R. Nürnberg, Finite element approximation of a phase field model for multicomponent surface diffusion, (in preparation) (2008).

[9] J. W. Barrett, R. Nürnberg, V. Styles, Finite element approximation of a phase field model for void electromigration, SIAM J. Numer. Anal. 42 (2) (2004) 738-772.

[10] K. A. Brakke, The surface evolver, Experiment. Math. 1 (2) (1992) 141-165.

[11] L. Bronsard, H. Garcke, B. Stoth, A multi-phase Mullins-Sekerka system: matched asymptotic expansions and an implicit time discretisation for the geometric evolution problem, Proc. Roy. Soc. Edinburgh Sect. A 128 (3) (1998) 481-506.

[12] L. Bronsard, F. Reitich, On three-phase boundary motion and the singular limit of a vector-valued Ginzburg-Landau equation, Arch. Rational Mech. Anal. 124 (4) (1993) $355-379$.

[13] J. W. Cahn, C. M. Elliott, A. Novick-Cohen, The Cahn-Hilliard equation with a concentration dependent mobility: motion by minus the Laplacian of the mean curvature, European J. Appl. Math. 7 (3) (1996) 287-301.

[14] A. Cañete, M. Ritoré, Least-perimeter partitions of the disk into three regions of given areas, Indiana Univ. Math. J. 53 (3) (2004) 883-904.

[15] B. Cipra, Why double bubbles form the way they do, Science 287 (5460) (2000) 1910-1912. 
[16] S. J. Cox, Calculations of the minimal perimeter for $N$ deformable cells of equal area confined in a circle, Phil. Mag. Letts. 86 (9) (2006) 569-578.

[17] S. J. Cox, F. Graner, Three-dimensional bubble clusters: Shape, packing, and growth rate, Phys. Rev. E 69 (3) (2004) 031409.

[18] S. J. Cox, F. Graner, M. F. Vaz, C. Monnereau-Pittet, N. Pittet, Minimal perimeter for $N$ identical bubbles in two dimensions: calculations and simulations, Phil. Mag. 83 (11) (2003) 1393-1406.

[19] D. de Fontaine, An analysis of clustering and ordering in multicomponent solid solutions-I. stability criteria, J. Phys. Chem. Solids 33 (1972) 297-310.

[20] C. M. Elliott, H. Garcke, On the Cahn-Hilliard equation with degenerate mobility, SIAM J. Math. Anal. 27 (1996) 404-423.

[21] C. M. Elliott, H. Garcke, Diffusional phase transitions in multicomponent systems with a concentration dependent mobility matrix, Phys. D 109 (1997) 242-256.

[22] F. Fischer, Four-bubble clusters and Menelaus' theorem, Amer. J. Phys. 70 (10) (2002) 986-991.

[23] J. Foisy, M. Alfaro, J. Brock, N. Hodges, J. Zimba, The standard double soap bubble in $\mathbb{R}^{2}$ uniquely minimizes perimeter, Pacific J. Math. 159 (1) (1993) 47-59.

[24] H. Garcke, K. Ito, Y. Kohsaka, Linearized stability analysis of stationary solutions for surface diffusion with boundary conditions, SIAM J. Math. Anal. 36 (4) (2005) 1031-1056.

[25] H. Garcke, B. Nestler, B. Stinner, F. Wendler, Allen-Cahn systems with volume constraints, Math. Models Methods Appl. Sci. 18 (8) (2008) 1347-1381.

[26] H. Garcke, B. Nestler, B. Stoth, On anisotropic order parameter models for multiphase systems and their sharp interface limits, Physica D 115 (1998) 87-108.

[27] H. Garcke, A. Novick-Cohen, A singular limit for a system of degenerate CahnHilliard equations, Adv. Differential Equations 5 (4-6) (2000) 401-434.

[28] J. Hass, M. Hutchings, R. Schlafly, The double bubble conjecture, Electron. Res. Announc. Amer. Math. Soc. 1 (3) (1995) 98-102.

[29] J. Hass, R. Schlafly, Double bubbles minimize, Ann. of Math. (2) 151 (2) (2000) $459-515$.

[30] S. Hildebrandt, A. Tromba, Mathematics and optimal form, Scientific American Library, New York, 1985.

[31] M. Hutchings, F. Morgan, M. Ritoré, A. Ros, Proof of the double bubble conjecture, Ann. of Math. (2) 155 (2) (2002) 459-489. 
[32] C. Isenberg, The science of soap films and soap bubbles, Tieto Ltd., Clevedon, 1978, with a foreword by George Porter.

[33] A. M. Kraynik, D. A. Reinelt, F. van Swol, Structure of random foam, Phys. Rev. Lett. 93 (20) (2004) 208301.

[34] G. Lawlor, F. Morgan, Paired calibrations applied to soap films, immiscible fluids, and surfaces or networks minimizing other norms, Pacific J. Math. 166 (1) (1994) $55-83$.

[35] H. G. Lee, J. Kim, A second-order accurate non-linear difference scheme for the N-component Cahn-Hilliard system, Physica A 387 (2008) 4787-4799.

[36] P. L. Lions, B. Mercier, Splitting algorithms for the sum of two nonlinear operators, SIAM J. Numer. Anal. 16 (1979) 964-979.

[37] F. Morgan, Soap bubbles in $\mathbb{R}^{2}$ and in surfaces, Pacific J. Math. 165 (2) (1994) $347-361$.

[38] F. Morgan, Immiscible fluid clusters in $\mathbb{R}^{2}$ and $\mathbb{R}^{3}$, Michigan Math. J. 45 (3) (1998) $441-450$.

[39] J. E. Morral, J. W. Cahn, Spinodal decomposition in ternary systems, Acta Metall. 19 (1971) 1037-1045.

[40] J. A. Nelder, R. Mead, A simplex method for function minimization, Comput. J. 7 (1965) 308-333.

[41] A. Schmidt, K. G. Siebert, Design of Adaptive Finite Element Software: The Finite Element Toolbox ALBERTA, vol. 42 of Lecture Notes in Computational Science and Engineering, Springer-Verlag, Berlin, 2005.

[42] J. M. Sullivan, F. Morgan, Open problems in soap bubble geometry, Internat. J. Math. 7 (6) (1996) 833-842.

[43] J. E. Taylor, The structure of singularities in soap-bubble-like and soap-film-like minimal surfaces, Ann. of Math. (2) 103 (3) (1976) 489-539.

[44] J. E. Taylor, Some mathematical challenges in materials science, Bull. Amer. Math. Soc. 40 (1) (2003) 69-87.

[45] B. White, Existence of least-energy configurations of immiscible fluids, J. Geom. Anal. 6 (1) (1996) 151-161.

[46] W. Wichiramala, Proof of the planar triple bubble conjecture, J. Reine Angew. Math. 567 (2004) 1-49.

[47] H.-K. Zhao, B. Merriman, S. Osher, L. Wang, Capturing the behavior of bubbles and drops using the variational level set approach, J. Comput. Phys. 143 (2) (1998) 495-518. 